\title{
General formalism for vibronic Hamiltonians in tetragonal symmetry and beyond
}

\author{
Riley J. Hickman, Robert A. Lang, and Tao Zeng \\ Department of Chemistry, Carleton University, \\ Ottawa, Ontario, K1S5B6, Canada
}

\begin{abstract}
We derive general expansion formulas in vibrational coordinates for all bimodal Jahn-Teller and pseudo-Jahn-Teller Hamiltonians in tetragonal symmetry. Symmetry information of all the vibronic Hamiltonian matrix elements is fully carried by up to only 4 eigenvalues of symmetry operators. This problem-to-eigenvalue reduction enables us to handle thousands of vibronic problems in one work. The derived bimodal formulas can be easily extended to cover problems with one or more than two vibrational modes. They lay a solid foundation for future vibronic coupling studies of tetragonal systems. More importantly, the efficient derivation can be applied to handle (pseudo)Jahn-Teller Hamiltonians for all problems with one principal symmetry axis.
\end{abstract}




\section{INTRODUCTION}

Polyatomic systems with a principal symmetry axis $\geq 3$-fold can exhibit non-accidental orbital degeneracy in electronic states. The degeneracy reversely distorts the structure to a lower symmetry and self-annihilates. This is the celebrated Jahn-Teller (JT) effect. ${ }^{1}$ It arises from the vibronic coupling between degenerate electronic states and non-totally-symmetric vibrations. Similar symmetry lowering also occurs for a non-degenerate state when its coupling with other states by non-totally-symmetric vibrations is strong enough, i.e., the pseudo-Jahn-Teller (pJT) effect. ${ }^{2}$ As the only source of spontaneous symmetry breaking in polyatomic systems, the JT and pJT effects play essential roles in physical chemistry and chemical physics; they are translated into a plain statement, "Nature tends to avoid degeneracies by means of symmetry breaking." 3 They have been subjects of a plethora of studies in the past eight decades. ${ }^{3-8}$

JT problems and the pJT problems that involve pseudo-degenerate electronic states are intrinsically nonadiabatic problems. A (p)JT Hamiltonian is expanded around a high symmetry structure in a set of degenerate/pseudo-degenerate electronic states and a set of relevant vibrational coordinates. A JT problem is generally labelled by $\Gamma \otimes\left(\gamma_{1}+\gamma_{2}+\cdots\right)$ and a pJT problem by $\left(\Gamma_{1}+\Gamma_{2}\right) \otimes\left(\gamma_{1}+\gamma_{2}+\cdots\right)$, with $\Gamma$ and $\gamma$ being irreducible representations (irreps) of electronic states and vibrations, respectively. Diabatic electronic states (diabats) are used to expand the Hamiltonian so that their matrix elements are smooth functions of vibrational coordinates and can be Taylor-expanded. ${ }^{9}$ In a diabatic representation, the nuclear kinetic operator is decoupled from electronic degrees of freedom, and the vibronic interaction is embedded in the variation of the diabatic electronic Hamiltonian matrix with respect to nuclear distortions. ${ }^{10-12}$ Correspondingly, in the text below, the term "Hamiltonian" means electronic Hamiltonian in diabatic representation unless further specified.

Traditionally, the Hamiltonian expansions in vibrational coordinates are truncated at the second order. ${ }^{7}$ However, a growing number of studies have revealed the limitations of the low-order Hamiltonians. For example, JT/pJT-active bending vibrations can bring nuclei close to each other and the resultant repulsion features positive anharmonicity. ${ }^{10,11,13,14}$ This is the so-called intramolecular collision. ${ }^{15}$ Also, when a JT/pJT problem involves bond dissociation, the diabatic potentials are anharmonic and require higher order expansions. ${ }^{16}$ 
High-order Hamiltonians are of concern to both theoreticians and experimentalists. They are needed to interpret complicated vibronic spectra. ${ }^{17,18}$ They are also needed to fit the spectra to obtain accurate empirical (p)JT parameters. ${ }^{19}$ Unfortunately, high-order expansion formulas of (p)JT Hamiltonians are largely unknown. This ignorance forces us to fit accurate numerical values of vibronic matrix elements using low-order polynomials, regrettably losing accuracy. Pioneering works to derive high-order (p)JT Hamiltonians were dedicated to textbook problems, e.g., the $E \otimes e$ problem in $C_{3 v}$ symmetry, the $T_{2} \otimes t_{2}$ and $T_{2} \otimes e$ problems in $T_{d}$ symmetry. ${ }^{10,11,20,21}$ These early case-specific attempts inspired us to develop general formalism for (p)JT Hamiltonians to arbitrary order. We have recently derived general formulas for $153(E+A) \otimes(e+a)$ problems in trigonal symmetry ${ }^{22}$ and 92 problems in tetrahedral and octahedral symmetries. ${ }^{23}$ These manually derived formulas feature the following advantages: (1) they are analytical. Up to an arbitrary order they are correct, complete, concise, and immune to numerical errors; (2) they clearly reveal connections between vibronic problems. For instance, they guide us to adapt a simulation program designed for one problem to another problem; (3) they allow us to circumvent conventional procedures of using Clebsch-Gordan coefficients ${ }^{24,25}$ or totally symmetric projection operators to construct Hamiltonians. The conventional methods are used in a case-by-case manner, which is laborious at high orders. Also, their results are subject to linear redundancy and numerical instability.

In this work, we manage to derive general Hamiltonian formulas for all bimodal (p)JT problems in tetragonal symmetry. Unimodal problems are special cases of bimodal problems with one set of vibrational coordinates set to 0 . Problems with more than two modes can be approximated as, e.g., $\Gamma \otimes\left(\gamma_{1}+\gamma_{2}+\gamma_{3}\right) \approx \Gamma \otimes\left(\gamma_{1}+\gamma_{2}\right)+\Gamma \otimes\left(\gamma_{1}+\gamma_{3}\right)+\Gamma \otimes\left(\gamma_{2}+\gamma_{3}\right)$, or more accurately treated following the same procedure here. These formulas are desired in studies of excitations and ionizations of systems in tetragonal symmetries ${ }^{26-28}$ as well as nonadiabatic dynamics/distortions around a tetragonal symmetric structure. ${ }^{29,30}$ This is our first, practical motivation. The second motivation is theory-wise. Tetragonal symmetry is the lowest axial symmetry (symmetry with one principal axis) that possesses all three $A$-, $B$-, and $E$-type irreps. As discussed at the end of the paper, the derivation here can be generalized to handle (p)JT problems in all axial symmetries, resulting in formalisms of similar simplicity and conciseness.

Our derivation is based on the old idea of "descent in symmetry", which was thoroughly 
discussed by Liehr more than half a century ago. ${ }^{31}$ By using this old method, we obtain a new formalism that will benefit future vibornic studies. For instance, what are the tenth order expansion formulas of the $E_{g} \otimes\left(e_{u}+b_{2 u}\right)$ problem in $D_{4 h}$ symmetry? Typical answers prior to this work might be: "they can be obtained following the route in Liehr's work"; "they can be obtained using the Wigner-Eckart Theorem and the Clebsch-Gordan coefficients". ${ }^{24,25}$ After this work, the answer is neat and clean:

$$
\begin{aligned}
& H_{+_{g}+g}=a_{2 I, 2 K}^{r, 4 k} w^{2 I} \rho^{|4 k|+2 K} \cos (4 k \phi) \text { with } 2 I+|4 k|+2 K=10 \\
& H_{+_{g}-g}=b_{2 I, 2 K}^{r, 4 k+2} w^{2 I} \rho^{|4 k+2|+2 K} e^{i(4 k+2) \phi} \text { with } 2 I+|4 k+2|+2 K=10
\end{aligned}
$$

For the twentieth order, we only need to change the summation of the powers to 20 . The arbitrariness of the expansion order is an improvement over previous derivations for (p)JT problems, ${ }^{10-12,31}$ which gave expansions up to high yet finite orders. In short, we have relieved future researchers in tetragonal (p)JT problems from deriving Hamiltonian formulas, and our formulas are highly programmable. These are the main practical merits of this study.

Please note that the derivation of high order (p)JT Hamiltonian formulas is not a trivial practice of knowledge in group theory and molecular symmetry. Incomplete or linearly redundant expansions are not rare in literatures; some examples are given in Section IV of Ref. 22. It requires a systematic and concise formalism to avoid these errors. In this work, we organize all bimodal (p)JT Hamiltonian formulas in one type of symmetry (tetragonal) into a few lookup tables. The resultant formalism is the most systematic and concise, and hence free from any error.

\section{SETTINGS, SYMBOLS, AND TERMINOLOGY}

There is an arbitrariness in orienting the two components of the degenerate $E$ irrep in tetragonal symmetry. We set the two components of an $E$ state and the two components of an $e$ vibrational mode so that they transform under $\hat{C}_{4}$ as

$$
\hat{C}_{4}|X\rangle=|Y\rangle ; \hat{C}_{4}|Y\rangle=-|X\rangle ; \hat{C}_{4} e_{x}=e_{y} ; \hat{C}_{4} e_{y}=-e_{x}
$$

Such a setting is exemplified in Figure 1(a). The $E$ components are transformed to be eigenstates of $\hat{C}_{4}$ :

$$
(|+\rangle|-\rangle)=(|X\rangle|Y\rangle) \frac{1}{\sqrt{2}}\left(\begin{array}{cc}
1 & 1 \\
i & -i
\end{array}\right) ; \hat{C}_{4}| \pm\rangle=\mp i| \pm\rangle
$$


$x$ and $y$ and their polar counterparts $\rho$ and $\phi(x=\rho \cos \phi ; y=\rho \sin \phi)$ are used as the coordinates of the $e_{x}$ and $e_{y}$ component modes. $z$ and $w$ are used as coordinates for $a$ and $b$ vibrations. $\alpha$ and $\beta$ are used to differentiate states or modes of the same irrep (e.g., see Figure 2). Henceforth, $\Gamma$ is used to represent the nondegenerate $A$ and $B$ states when the two are grouped together in discussion, and $v$ for $a$ and $b$ modes' coordinates.

(a) $C$

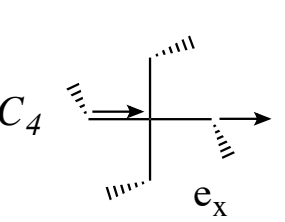

(b) $S_{4}$

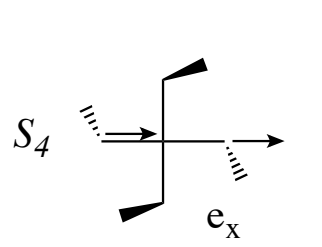

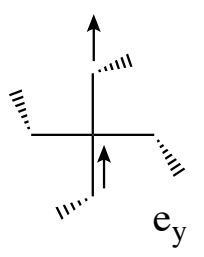
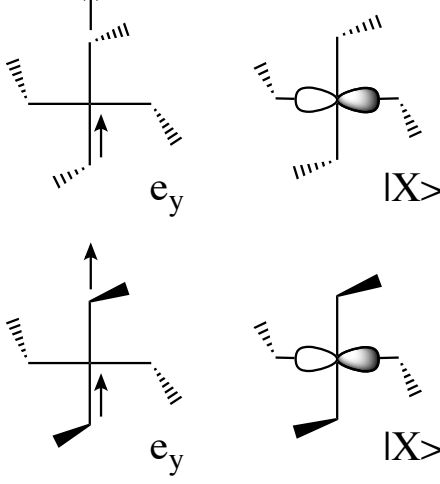

(c) $C_{4 v}$

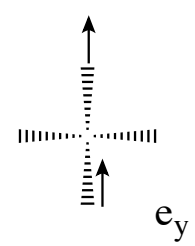

(d)

$\mathrm{D}_{4}$
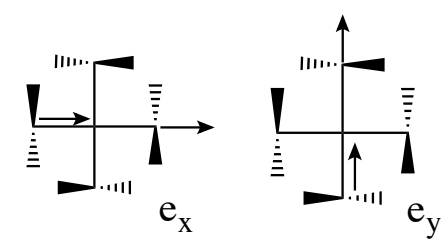

(e) $D_{2 d}$
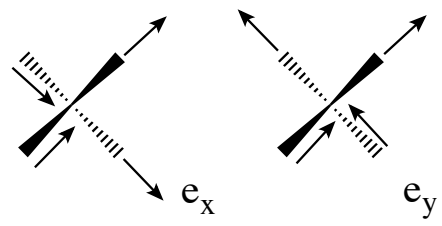
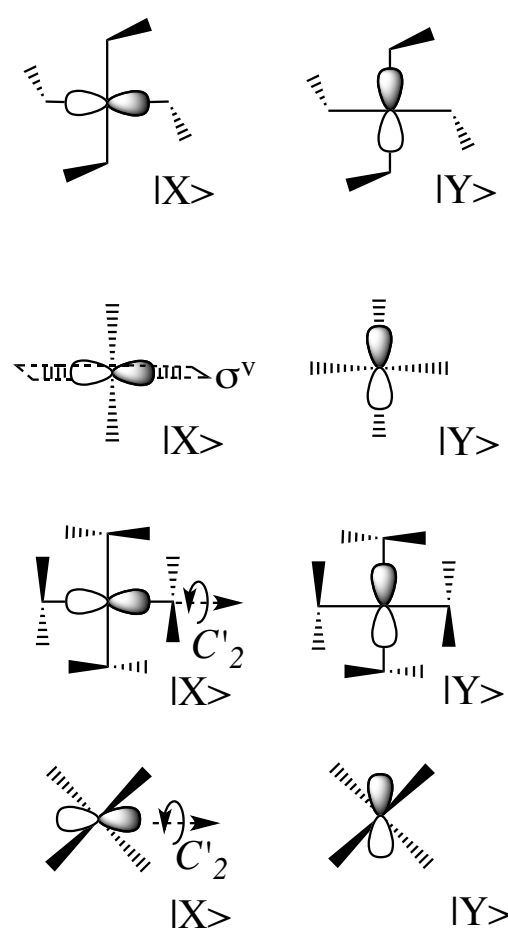

$\mid X>$
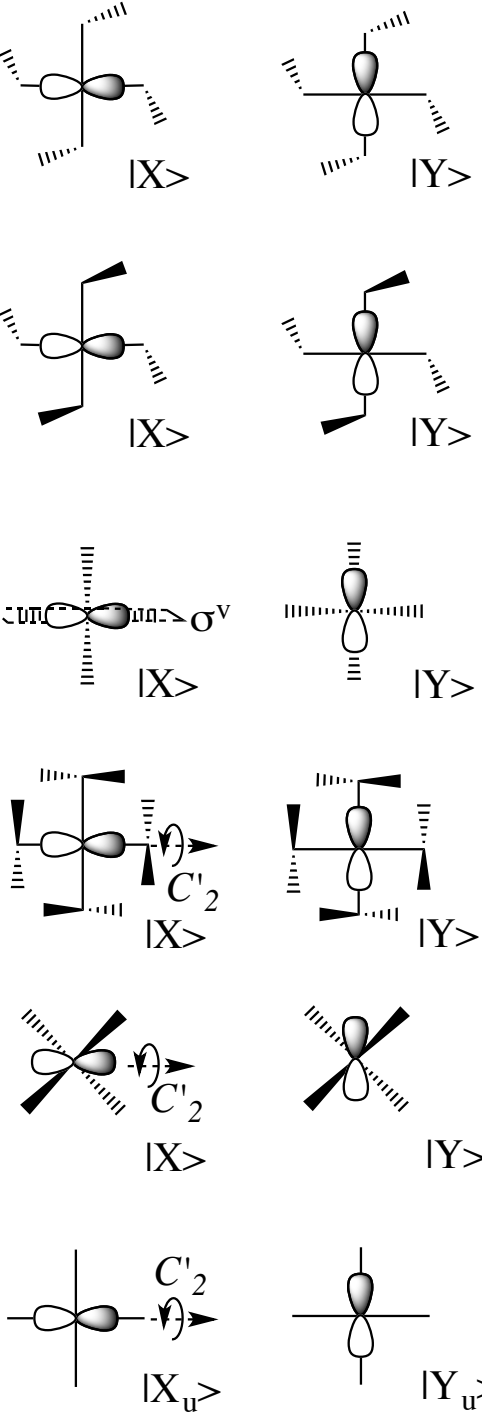

Y>

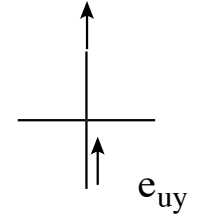

$\left|X_{\mathrm{u}}\right\rangle$ $\mid Y_{\mathrm{u}}>$

FIG. 1. Examples of $E$ components and $e$ components settings in 6 tetragonal point groups.

Both the root-branch and the modularized approaches are employed in the following derivation. The essence of the root-branch approach is to first derive the expansion formulas (root formulas) for the lowest symmetry $\left(C_{4}\right)$ problems, and then to impose constraints on the root formulas to obtain expansions for higher symmetry $\left(C_{4 v}, C_{4 h}\right.$, etc.) problems 
(branch formulas).The essence of this approach is the "mathematical inheritance" that the high symmetry problems obtain from the low symmetry problems. ${ }^{31}$ Please note the unidirectional inheritance from low to high symmetry. If we derive low symmetry formulas from high symmetry formulas, e.g., from $D_{3 h}$ to $C_{3 v}$ and $C_{3 h}$, the results are prone to errors. Within each symmetry, we first handle interterm couplings between states from two term symbols (i.e., pJT interaction). Formulas of intraterm couplings between states of the same term symbol (i.e., JT interaction) are easily obtained from interterm results. The difference between the two types of problems is illustrated in Figure 2. In the text below, symbols like $(E+B) \otimes(e+a)(E \otimes(e+a))$ are reserved for interterm (intraterm) problems. Among

the seven tetragonal point groups: $C_{4}, S_{4}, C_{4 v}, D_{4}, D_{2 d}, C_{4 h}$, and $D_{4 h}$, the first two are isomorphic, and so are the third to the fifth. Therefore, we only need to derive Hamiltonian formulas for the $C_{4}, C_{4 v}, C_{4 h}$, and $D_{4 h}$ symmetries.

$$
\left(\left(\left|E^{\alpha}\right\rangle\right)_{1 \times 2},\left(\left|E^{\beta}\right\rangle\right)_{1 \times 2}\right)\left(\begin{array}{cc}
H_{2 \times 2}^{\alpha \alpha} & H_{2 \times 2}^{\alpha \beta} \\
\left(H_{2 \times 2}^{\alpha \beta}\right)^{\dagger} & H_{2 \times 2}^{\beta \beta}
\end{array}\right)\left(\begin{array}{l}
\left(\left\langle E^{\alpha}\right|\right)_{2 \times 1} \\
\left(\left\langle E^{\beta}\right|\right)_{2 \times 1}
\end{array}\right)
$$

FIG. 2. The matrix product form of a vibronic coupling Hamiltonian that involves two $E$ term symbols denoted by $\alpha$ and $\beta$. The green and blue diagonal blocks of the $H$ matrix give the intraterm couplings, and the red off-diagonal blocks give the interterm coupling.

\section{DERIVATION AND RESULTS}

\section{A. $C_{4}$ and $S_{4}$ Problems.}

The essence of the modularized approach in deriving (p)JT Hamiltonians is to consider the electronic and the vibrational parts of a vibronic problem separately. ${ }^{23}$ We first consider the electronic parts of interterm problems in $C_{4}$ symmetry. We start with the $(E+\Gamma)$-type problems, which have a Hamiltonian

$$
\hat{H}=(|+\rangle\langle\Gamma|+| \Gamma\rangle\langle-|) H_{+\Gamma}+h c
$$

$h c$ stands for the hermitian conjugate of the explicitly written part. The time-reversal symmetry has been employed to derive $H_{-\Gamma}=H_{+\Gamma}^{*}$. An external magnetic field is not 
considered in this work and thus all problems are time-reversal-symmetric. For all problems below, when their Hamiltonians are first written, the time-reversal symmetry has been imposed to retain only unique matrix elements. The fundamental requirement of a (p)JT Hamiltonian is its invariance with respect to all symmetry operators. Acting $\hat{C}_{4}$ on Eq. 4 ,

$$
\hat{C}_{4} \hat{H} \hat{C}_{4}^{-1}=\mp i(|+\rangle\langle\Gamma|+| \Gamma\rangle\langle-|) \hat{C}_{4} H_{+\Gamma}+h c .
$$

In this subsection, the top (bottom) symbols of $\mp$ and \pm are taken when $\Gamma=A(B)$. Comparing Eqs. 4 and 5, we see that $\hat{C}_{4} H_{+\Gamma}= \pm i H_{+\Gamma}$ is needed for a $\hat{C}_{4}$-invariant $\hat{H}$.

The Hamiltonian of the $(E+E)$-type problems reads

$$
\begin{array}{r}
\hat{H}=\left(\left|+_{\alpha}\right\rangle\left\langle+_{\beta}|+|-_{\beta}\right\rangle\left\langle-_{\alpha}\right|\right) H_{+_{\alpha}+_{\beta}}+\left(\left|+_{\alpha}\right\rangle\left\langle-_{\beta}|+|+_{\beta}\right\rangle\left\langle-_{\alpha}\right|\right) H_{+_{\alpha}-\beta}+h c ;(6) \\
\hat{C}_{4} \hat{H} \hat{C}_{4}^{-1}=\left(\left|+_{\alpha}\right\rangle\left\langle+_{\beta}|+|-_{\beta}\right\rangle\left\langle-_{\alpha}\right|\right) \hat{C}_{4} H_{+_{\alpha}{ }_{\beta} \beta}-\left(\left|+_{\alpha}\right\rangle\left\langle-_{\beta}|+|+{ }_{\beta}\right\rangle\left\langle-_{\alpha}\right|\right) \hat{C}_{4} H_{+_{\alpha}-\beta}+h c .(7)
\end{array}
$$

$\hat{C}_{4} \hat{H} \hat{C}_{4}^{-1}=\hat{H}$ requires $\hat{C}_{4} H_{+_{\alpha}{ }_{\beta}}=H_{+_{\alpha}+_{\beta}}$ and $\hat{C}_{4} H_{+_{\alpha}-\beta}=-H_{+_{\alpha}-\beta}$. For the $(\Gamma+\Gamma)$-type problems with two identical $\Gamma$ s,

$$
\hat{H}=\left(\left|\Gamma_{\alpha}\right\rangle\left\langle\Gamma_{\beta}|+| \Gamma_{\beta}\right\rangle\left\langle\Gamma_{\alpha}\right|\right) H_{\Gamma_{\alpha} \Gamma_{\beta}}^{r} ; \hat{C}_{4} \hat{H} \hat{C}_{4}^{-1}=\left(\left|\Gamma_{\alpha}\right\rangle\left\langle\Gamma_{\beta}|+| \Gamma_{\beta}\right\rangle\left\langle\Gamma_{\alpha}\right|\right) \hat{C}_{4} H_{\Gamma_{\alpha} \Gamma_{\beta}}^{r} .
$$

$\hat{C}_{4} \hat{H} \hat{C}_{4}^{-1}=\hat{H}$ requires $\hat{C}_{4} H_{\Gamma_{\alpha} \Gamma_{\beta}}^{r}=H_{\Gamma_{\alpha} \Gamma_{\beta}}^{r}$. The superscript $r$ indicates a real matrix element, which results from the $|\Gamma\rangle$ states being real. Finally, for the $(A+B)$-type problems,

$$
\hat{H}=(|A\rangle\langle B|+| B\rangle\langle A|) H_{A B}^{r} ; \hat{C}_{4} \hat{H} \hat{C}_{4}^{-1}=-(|A\rangle\langle B|+| B\rangle\langle A|) \hat{C}_{4} H_{A B}^{r} .
$$

$\hat{C}_{4} \hat{H} \hat{C}_{4}^{-1}=\hat{H}$ requires $\hat{C}_{4} H_{A B}^{r}=-H_{A B}^{r}$. The electronic parts of all interterm problems in $C_{4}$ symmetry have been considered. It is clear that to have the interterm Hamiltonians symmetry-adapted, their independent matrix elements $\left(H_{i j} \mathrm{~s}\right)$ need to be $\hat{C}_{4}$-eigenfunctions, with their eigenvalues $\left(\chi^{C_{4}} \mathrm{~s}\right)$ summarized in Table 1 . At this moment, the subscripts $k, l$, $p, q$ and all other $\sigma_{v^{-}}$and $I$-related quantities in the table shall be ignored.

There are three types of intraterm problems in $C_{4}$ symmetry. For the $A$ - and $B$-type problems, it is straightforward to see that the lone, real matrix element $H_{\Gamma \Gamma}^{r}$ is $\hat{C}_{4}$-invariant $\left(\chi^{C_{4}}=1\right)$. For the E-type problems,

$$
\hat{H}=(|+\rangle\langle+|+|-\rangle\langle-|) H_{++}^{r}+|+\rangle\left\langle-\left|H_{+-}+\right|-\right\rangle\langle+| H_{+-}^{*} .
$$

Since $|+\rangle\langle+|$ and $|+\rangle\langle-|$ transform just like $\left|+_{\alpha}\right\rangle\left\langle+_{\beta}\right|$ and $\left|+_{\alpha}\right\rangle\left\langle-_{\beta}\right|$ under $\hat{C}_{4}, H_{++}^{r}$ is a special case of $H_{+_{\alpha}{ }_{\beta}}$ being real, and $H_{+-}$shares the same $\chi^{C_{4}}$ with $H_{+_{\alpha}-\beta}$. All the intraterm $\chi^{C_{4}}$ s are included in Table 1. 
TABLE 1. The eigenvalues of symmetry operators of the independent elements in tetragonal vibronic Hamiltonian matrices. The $\hat{\sigma}_{v}$-eigenvalues are given for the real and imaginary parts of the matrix elements separately. The heading $(A+A) /(B+B)$ means the matrix elements underneath are relevant to the $(A+A)$ and $(B+B)$ problems, etc. $k$ and $\ell$ stand for possible $1 / 2$ subscripts for $\Gamma, p$ and $q$ stand for possible $g / u$ subscripts.

\begin{tabular}{|c|c|c|}
\hline & $(A+A) /(B+B) / A / B$ & $(A+B)$ \\
\hline$i j: \chi^{C_{4}},\left(\chi_{R e}^{\sigma_{v}}, \chi_{I m}^{\sigma_{v}}\right), \chi^{I}$ & $\begin{array}{c}A_{k p} A_{\ell q} / B_{k p} B_{\ell q}: 1,\left((-1)^{\delta_{k \ell}+1}, 0\right),(-1)^{\delta_{p q}+1} \\
\Gamma_{k p} \Gamma_{k p}: 1,(1,0), 1\end{array}$ & $A_{k p} B_{\ell q}:-1,\left((-1)^{\delta_{k \ell}+1}, 0\right),(-1)^{\delta_{p q}+1}$ \\
\hline & $(E+A)$ & $(E+B)$ \\
\hline \multirow{2}{*}{$i j: \chi^{C_{4}},\left(\chi_{R e}^{\sigma_{v}}, \chi_{I m}^{\sigma_{v}}\right), \chi^{I}$} & $+{ }_{p} A_{k q}: i,\left((-1)^{\delta_{k 2}},(-1)^{\delta_{k 1}}\right),(-1)^{\delta_{p q}+1}$ & $+{ }_{p} B_{k q}:-i,\left((-1)^{\delta_{k 2}},(-1)^{\delta_{k 1}}\right),(-1)^{\delta_{p q}+1}$ \\
\hline & $(E+E)$ & $E$ \\
\hline$i j: \chi^{C_{4}},\left(\chi_{R e}^{\sigma_{v}}, \chi_{I m}^{\sigma_{v}}\right), \chi^{I}$ & $\begin{array}{l}+_{\alpha p}+\beta_{\beta q}: 1,(1,-1),(-1)^{\delta_{p q}+1} \\
+_{\alpha p}-\beta q:-1,(1,-1),(-1)^{\delta_{p q}+1}\end{array}$ & $\begin{array}{c}++: 1,(1,0), 1 \\
+-:-1,(1,-1), 1\end{array}$ \\
\hline
\end{tabular}

The next step is to construct polynomials of vibrational coordinates that are $\hat{C}_{4^{-}}$ eigenfunctions. Those polynomials are modules to expand the matrix elements with the corresponding $\hat{C}_{4}$-eigenvalues. The coordinates transform under $\hat{C}_{4}$ as

$$
\hat{C}_{4} f(z, w, \rho, \phi)=f\left(z,-w, \rho, \phi-\frac{\pi}{2}\right) .
$$

We use projection operators of the three irreps of the $C_{4}$ point group to construct the eigenfunctions. Let's take the $(e+b)$ vibrational part as an example. A function of a $b$ coordinate and a set of $e$ coordinates can in general be expanded as

$$
f(w, \rho, \phi)=a_{m, n, K} w^{m} \rho^{|n|+2 K} e^{i n \phi} ; m, K=0,1,2, \cdots ; n=\cdots,-1,0,1, \cdots
$$

$a_{m, n, K}$ stands for expansion coefficient and is in general complex. Einstein's convention of summing over duplicate indices is followed in all expansion formulas. The $|n|+2 K$ power of $\rho$ is a necessary constraint to ensure that the $e$ coordinates part can be written as a Taylor expansion in $x$ and $y$. The full ranges of summation indices guarantee the completeness of this expansion. Throughout this paper, the indices that appear within an absolute value symbol take all integer values; the others take nonnegative integer values. 
Acting the projection operators of the $A-, B-$, and $E_{+1}$-irreps on $f$ gives $\hat{C}_{4}$-eigenfunctions with eigenvalues 1, -1 , and $i$ (see details in Eqs. S1 to S3 in the Electronic Supporting Information (ESI)):

$$
\begin{aligned}
f^{(1)}(w, \rho, \phi) & =a_{I, 2 n, K} w^{\bmod (n, 2)+2 I} \rho^{|2 n|+2 K} e^{i 2 n \phi} ; \\
f^{(-1)}(w, \rho, \phi) & =a_{I, 2 n, K} w^{\bmod (n, 2)+2 I+1} \rho^{|2 n|+2 K} e^{i 2 n \phi} ; \\
f^{(i)}(w, \rho, \phi) & =a_{I, 2 n-1, K} w^{\bmod (n, 2)+2 I} \rho^{|2 n-1|+2 K} e^{i(2 n-1) \phi} .
\end{aligned}
$$

$f^{(i) *}(w, \rho, \phi)$ belongs to the $E_{-1}$-irrep and has the $\hat{C}_{4}$-eigenvalue $-i$. These $(e+b)$ eigenexpansions are complete because: (1) the original expansion in Eq. 12 is complete and it can be partitioned into contributions exclusively belonging to the four irreps; (2) the action of the projection operator of each of the irreps does not discard any components that belong to that irrep.

Following the same procedure, $\hat{C}_{4}$-eigen-expansions of all bimodal vibrational parts have been derived and summarized in Tables 2 to 4 . In these tables, "na" means "not applicable", i.e., there is no expansion in the corresponding vibrational coordinates that has the specific $\hat{C}_{4}$-eigenvalue. For the same two reasons mentioned above, the expansions are all complete. Because expansion formulas with the $\hat{C}_{4}$-eigenvalue $-i$ are simply complex conjugates of those with eigenvalue $i$, they are not given. In these tables, expansion coefficients are complex unless they are denoted by superscripts $r$ or $i$, which are real and stem from the respective real and imaginary parts of their complex parents. It is important to allow the coefficients to be complex in deriving vibronic Hamiltonians. As discussed in Section IV.B of Ref. 22, erroneous formulas can be obtained for trigonal (p)JT problems if the coefficients are assumed to be real without justification. Given the tabulated modules, we can easily write down the Hamiltonian expansion formulas for any of the 36 (6 electronic $\times 6$ vibrational parts) interterm and $18(3 \times 6)$ intraterm problems. For instance, for the $(E+E) \otimes(e+b)$ problem, we simply select the $(e+b)$ formulas in Tables 2 and 3 for $H_{+_{\alpha}+_{\beta}}$ and $H_{+_{\alpha}-\beta}$, respectively, matching the matrix elements' $\hat{C}_{4}$-eigenvalues 1 and -1 in Table 1. The modularized approach has simplified the derivation of $C_{4}(\mathrm{p}) \mathrm{JT}$ Hamiltonians to a matching game. We make no attempt to claim innovation in using this convenient approach. Its central idea was mentioned, e.g., in Ref. 31 (see note 9 there).

For real matrix elements, e.g., $H_{A_{\alpha} A_{\beta}}^{r}$ and $H_{A B}^{r}$, one just takes the real parts of the appropriate expansions. This is because all the real matrix elements have 1 or $-1 \hat{C}_{4^{-}}$ 
TABLE 2. Expansion formulas for $\hat{C}_{4}$-eigenfunctions of the bimodal vibrational coordinates with eigenvalue 1 .

\begin{tabular}{|c|c|}
\hline$(a+a)$ & $a_{I_{1}, I_{2}}^{r} z_{\alpha}^{I_{1}} z_{\beta}^{I_{2}}+i a_{I_{3}, I_{4}}^{i} z_{\alpha}^{I_{3}} z_{\beta}^{I_{4}}$ \\
\hline \multicolumn{2}{|r|}{$(a+b) a_{I_{1}, 2 J}^{r} z^{I_{1}} w^{2 J}+i a_{I_{2}, 2 J}^{i} z^{I_{2}} w^{2 J}$} \\
\hline$(b+b)$ & $\begin{array}{l}a_{2 J_{1}+1,2 J_{2}+1}^{r} w_{\alpha}^{2 J_{1}+1} w_{\beta}^{2 J_{2}+1}+i a_{2 J_{1}+1,2 J_{2}+1}^{i} w_{\alpha}^{2 J_{1}+1} w_{\beta}^{2 J_{2}+1} \\
+a_{2 J_{1}, 2 J_{2}}^{r} w_{\alpha}^{2 J_{1}} w_{\beta}^{2 J_{2}}+i a_{2 J_{1}, 2 J_{2}}^{i} w_{\alpha}^{2 J_{1}} w_{\beta}^{2 J_{2}}\end{array}$ \\
\hline$(e+a)$ & $\begin{array}{l}a_{I, 2 K}^{4 m} z^{I} \rho^{|4 m|+2 K} e^{i 4 m \phi}=\rho^{|4 m|+2 K}\left[a_{I_{1}, 2 K}^{r, 4 m} z^{I_{1}} \cos (4 m \phi)-a_{I_{2}, 2 K}^{i, 4 m} z^{I_{2}} \sin (4 m \phi)\right. \\
\left.+i a_{I_{1}, 2 K}^{r, 4 m} z^{I_{1}} \sin (4 m \phi)+i a_{I_{2}, 2 K}^{i, 4 m} z^{I_{2}} \cos (4 m \phi)\right]\end{array}$ \\
\hline$(e+b)$ & $\begin{array}{l}a_{2 I, 2 K}^{2 m} w^{\bmod (m, 2)+2 I} \rho^{|2 m|+2 K} e^{i 2 m \phi}=w^{\bmod (m, 2)+2 I} \rho^{|2 m|+2 K}\left[a_{2 I, 2 K}^{r, 2 m} \cos (2 m \phi)\right. \\
\left.-a_{2 I, 2 K}^{i, 2 m} \sin (2 m \phi)+i a_{2 I, 2 K}^{r, 2 m} \sin (2 m \phi)+i a_{2 I, 2 K}^{i, 2 m} \cos (2 m \phi)\right]\end{array}$ \\
\hline$(e+e)$ & $\begin{array}{l}a_{2 K_{1}, 2 K_{2}}^{m_{1}, 4 n} \rho_{\alpha}^{\left|m_{1}\right|+2 K_{1}} \rho_{\beta}^{\left|4 n-m_{1}\right|+2 K_{2}} e^{i\left(m_{1} \phi_{\alpha}+\left(4 n-m_{1}\right) \phi_{\beta}\right)}=\rho_{\alpha}^{\left|m_{1}\right|+2 K_{1}} \rho_{\beta}^{\left|4 n-m_{1}\right|+2 K_{2}} \\
{\left[\left(a_{2 K_{1}, 2 K_{2}}^{r, m_{1}, 4 n} \cos \left(m_{1} \phi_{\alpha}+\left(4 n-m_{1}\right) \phi_{\beta}\right)-a_{2 K_{1}, 2 K_{2}}^{i, m_{1}, 4 n} \sin \left(m_{1} \phi_{\alpha}+\left(4 n-m_{1}\right) \phi_{\beta}\right)\right)\right.} \\
+i\left(a_{2 K_{1}, 2 K_{2}}^{r, m_{1}, 4 n} \sin \left(m_{1} \phi_{\alpha}+\left(4 n-m_{1}\right) \phi_{\beta}\right)+a_{2 K_{1}, 2 K_{2}}^{i, m_{1}, 4 n} \cos \left(m_{1} \phi_{\alpha}+\left(4 n-m_{1}\right) \phi_{\beta}\right)\right)\end{array}$ \\
\hline
\end{tabular}

eigenvalues, and they can be expanded only in basis sets of correspondingly $A$ or $B$ irrep. Basis sets of $A$ and $B$ irreps can always be chosen real. The real and imaginary parts of a function with $1(-1) \hat{C}_{4}$-eigenvalue are expanded in the same complete real $A$-irrep $(B$ irrep) basis set but with real and imaginary coefficients, respectively. Therefore, the real and imaginary parts are respectively $\hat{C}_{4}$-eigenfunctions with eigenvalue $1(-1)$; when taking only the real parts, the eigenvalue and the completeness are not lost.

The formulas in Tables 2 to 4 also apply to the $54(36+18)$ Hamiltonians in $S_{4}$ symmetry, as long as the $E$ and $e$ settings follow Eq. 2, but with $\hat{C}_{4}$ being replaced by $\hat{S}_{4}$ (e.g., Figure 1(b)). These tables provide root formulas for the following derivation for higher symmetries (the branches).

\section{B. $\quad C_{4 v}, D_{4}$, and $D_{2 d}$ Problems.}

We select the $\hat{\sigma}_{v}$ that conserves $|X\rangle$ and $e_{x}$ but flip $|Y\rangle$ and $e_{y}$ as the representative reflection operator of $C_{4 v}$ symmetry (e.g., Figure 1(c)). It transforms the electronic states 
TABLE 3. Expansion formulas for $\hat{C}_{4}$-eigenfunctions of the bimodal vibrational coordinates with eigenvalue -1 .

\begin{tabular}{|c|c|}
\hline$(a+a)$ & na \\
\hline$(a+b)$ & $b_{I_{1}, 2 J+1}^{r} z^{I_{1}} w^{2 J+1}+i b_{I_{2}, 2 J+1}^{i} z^{I_{2}} w^{2 J+1}$ \\
\hline$(b+b)$ & $b_{2 J_{1}+1,2 J_{2}}^{r} w_{\alpha}^{2 J_{1}+1} w_{\beta}^{2 J_{2}}+i b_{2 J_{1}+1,2 J_{2}}^{i} w_{\alpha}^{2 J_{1}+1} w_{\beta}^{2 J_{2}}+b_{2 J_{1}, 2 J_{2}+1}^{r} w_{\alpha}^{2 J_{1}} w_{\beta}^{2 J_{2}+1}+i b_{2 J_{1}, 2 J_{2}+1}^{i} w_{\alpha}^{2 J_{1}} w_{\beta}^{2 J_{2}+1}$ \\
\hline$(e+a)$ & $\begin{array}{l}b_{I, 2 K}^{4 n+2} z^{I} \rho^{|4 n+2|+2 K} e^{i(4 n+2) \phi}=\rho^{|4 n+2|+2 K}\left[b_{I_{1}, 2 K}^{r, 4 n+2} z^{I_{1}} \cos ((4 n+2) \phi)-b_{I_{2}, 2 K}^{i, 4 n+2} z^{I_{2}} \sin ((4 n+2) \phi)\right. \\
\left.+i b_{I_{1}, 2 K}^{r, 4 n+2} z^{I_{1}} \sin ((4 n+2) \phi)+i b_{I_{2}, 2 K}^{i, 4 n+2} z^{I_{2}} \cos ((4 n+2) \phi)\right]\end{array}$ \\
\hline$(e+b)$ & $\begin{array}{l}b_{2 I, 2 K}^{2 m} w^{\bmod (m, 2)+2 I+1} \rho^{|2 m|+2 K} e^{i 2 m \phi}=w^{\bmod (m, 2)+2 I+1} \rho^{|2 m|+2 K}\left[b_{2 I, 2 K}^{r, 2 m} \cos (2 m \phi)-b_{2 I, 2 K}^{i, 2 m} \sin (2 m \phi)\right. \\
\left.+i b_{2 I, 2 K}^{r, 2 m} \sin (2 m \phi)+i b_{2 I, 2 K}^{i, 2 m} \cos (2 m \phi)\right]\end{array}$ \\
\hline$(e+e)$ & $\begin{array}{l}b_{2 K_{1}, 2 K_{2}}^{m_{1}, 4 n+2} \rho_{\alpha}^{\left|m_{1}\right|+2 K_{1}} \rho_{\beta}^{\left|4 n+2-m_{1}\right|+2 K_{2}} e^{i\left(m_{1} \phi_{\alpha}+\left(4 n+2-m_{1}\right) \phi_{\beta}\right)}=\rho_{\alpha}^{\left|m_{1}\right|+2 K_{1}} \rho_{\beta}^{\left|4 n+2-m_{1}\right|+2 K_{2}} \\
{\left[\left(b_{2 K_{1}, 2 K_{2}}^{r, m_{1}, 4 n+2} \cos \left(m_{1} \phi_{\alpha}+\left(4 n+2-m_{1}\right) \phi_{\beta}\right)-b_{2 K_{1}, 2 K_{2}}^{i, m_{1}, 4 n+2} \sin \left(m_{1} \phi_{\alpha}+\left(4 n+2-m_{1}\right) \phi_{\beta}\right)\right)\right.} \\
\left.+i\left(b_{2 K_{1}, 2 K_{2}}^{r, m_{1}, 4 n+2} \sin \left(m_{1} \phi_{\alpha}+\left(4 n+2-m_{1}\right) \phi_{\beta}\right)+b_{2 K_{1}, 2 K_{2}}^{i, m_{1}, 4 n+2} \cos \left(m_{1} \phi_{\alpha}+\left(4 n+2-m_{1}\right) \phi_{\beta}\right)\right)\right]\end{array}$ \\
\hline
\end{tabular}

and the vibrational coordinates as

$$
\hat{\sigma}_{v}\left(|+\rangle,|-\rangle,\left|\Gamma_{1}\right\rangle,\left|\Gamma_{2}\right\rangle\right)=\left(|-\rangle,|+\rangle,\left|\Gamma_{1}\right\rangle,-\left|\Gamma_{2}\right\rangle\right) ; \hat{\sigma}_{v} f\left(\rho, \phi, v_{1}, v_{2}\right)=f\left(\rho,-\phi, v_{1},-v_{2}\right) .
$$

The subscripts 1 and 2 determine the sign of $\Gamma$ states and $v$ coordinates under the reflection. Henceforth, the $1 / 2$ subscripts are labelled by $k$ and $\ell$. We group the $\left(A_{k}+A_{\ell}\right)^{-},\left(A_{k}+B_{\ell}\right)^{-}$, and $\left(B_{k}+B_{\ell}\right)$-type interterm problems in deriving extra requirements on their Hamiltonian matrix elements in $C_{4 v}$ symmetry:

$$
\hat{H}=\left(\left|\Gamma_{k}\right\rangle\left\langle\Gamma_{\ell}|+| \Gamma_{\ell}\right\rangle\left\langle\Gamma_{k}\right|\right) H_{\Gamma_{k} \Gamma_{\ell}}^{r} ; \hat{\sigma}_{v} \hat{H} \hat{\sigma}_{v}^{-1}=(-1)^{\delta_{k \ell}+1}\left(\left|\Gamma_{k}\right\rangle\left\langle\Gamma_{\ell}|+| \Gamma_{\ell}\right\rangle\left\langle\Gamma_{k}\right|\right) \hat{\sigma}_{v} H_{\Gamma_{k} \Gamma_{\ell}}^{r} \cdot(17
$$

$\hat{\sigma}_{v} \hat{H} \hat{\sigma}_{v}^{-1}=\hat{H}$ requires $\hat{\sigma}_{v} H_{\Gamma_{k} \Gamma_{\ell}}^{r}=(-1)^{\delta_{k \ell}+1} H_{\Gamma_{k} \Gamma_{\ell}}^{r}$, where the Kronecker delta $\delta_{k \ell}$ concisely expresses the $\hat{\sigma}_{v}$-eigenvalues of the $\left|\Gamma_{k}\right\rangle\left\langle\Gamma_{\ell}\right|$ dyad and $H_{\Gamma_{k} \Gamma_{\ell}}^{r}$. For the $\left(E+\Gamma_{k}\right)$-type problems,

$$
\hat{H}=\left(|+\rangle\left\langle\Gamma_{k}|+| \Gamma_{k}\right\rangle\langle-|\right) H_{+\Gamma_{k}}+h c ; \hat{\sigma}_{v} \hat{H} \hat{\sigma}_{v}^{-1}=(-1)^{\delta_{k 2}}\left(|-\rangle\left\langle\Gamma_{k}|+| \Gamma_{k}\right\rangle\langle+|\right) \hat{\sigma}_{v} H_{+\Gamma_{k}}+h c .
$$


TABLE 4. Expansion formulas for $\hat{C}_{4}$-eigenfunctions of the bimodal vibrational coordinates with eigenvalue $i$.

\begin{tabular}{|c|c|}
\hline$+\gamma)^{\dagger}$ & \\
\hline$(e+a)$ & $\begin{array}{l}c_{I, 2 K}^{4 n+1} z^{I} \rho^{|4 n-1|+2 K} e^{i(4 n-1) \phi}=\rho^{|4 n-1|+2 K}\left[c_{I_{1}, 2 K}^{r, 4 n-1} z^{I_{1}} \cos ((4 n-1) \phi)-c_{I_{2}, 2 K}^{i, 4 n-1} z^{I_{2}} \sin ((4 n-1) \phi)\right. \\
+i \rho^{|4 n-1|+2 K}\left[c_{I_{1}, 2 K}^{r, 4 n-1} z^{I_{1}} \sin ((4 n-1) \phi)+c_{I_{2}, 2 K}^{i, 4 n-1} z^{I_{2}} \cos ((4 n-1) \phi)\right]\end{array}$ \\
\hline$(e+b)$ & $\begin{array}{l}c_{2 I, 2 K}^{2 n-1} w^{\bmod (n, 2)+2 I} \rho^{|2 n-1|+2 K} e^{i(2 n-1) \phi} \\
=w^{\bmod (n, 2)+2 I} \rho^{|2 n-1|+2 K}\left[c_{2 I, 2 K}^{r, 2 n-1} \cos ((2 n-1) \phi)-c_{2 I, 2 K}^{i, 2 n-1} \sin ((2 n-1) \phi)\right] \\
+i w^{\bmod (n, 2)+2 I} \rho^{|2 n-1|+2 K}\left[c_{2 I, 2 K}^{r, 2 n-1} \sin ((2 n-1) \phi)+c_{2 I, 2 K}^{i, 2 n-1} \cos ((2 n-1) \phi)\right]\end{array}$ \\
\hline$(e+e)$ & $\begin{array}{l}c_{2 K_{1}, 2 K_{2}}^{m_{1}, 4 n-1} \rho_{\alpha}^{\left|m_{1}\right|+2 K_{1}} \rho_{\beta}^{\left|4 n-1-m_{1}\right|+2 K_{2}} e^{i\left(m_{1} \phi_{\alpha}+\left(4 n-1-m_{1}\right) \phi_{\beta}\right)}=\rho_{\alpha}^{\left|m_{1}\right|+2 K_{1}} \rho_{\beta}^{\left|4 n-1-m_{1}\right|+2 K_{2}} \\
{\left[\left(c_{2 K_{1}, 2 K_{2}}^{r, m_{1}, 4 n-1} \cos \left(m_{1} \phi_{\alpha}+\left(4 n-1-m_{1}\right) \phi_{\beta}\right)-c_{2 K_{1}, 2 K_{2}}^{i, m_{1}, 4 n-1} \sin \left(m_{1} \phi_{\alpha}+\left(4 n-1-m_{1}\right) \phi_{\beta}\right)\right)\right.} \\
\left.+i\left(c_{2 K_{1}, 2 K_{2}}^{r, m_{1}, 4 n-1} \sin \left(m_{1} \phi_{\alpha}+\left(4 n-1-m_{1}\right) \phi_{\beta}\right)+c_{2 K_{1}, 2 K_{2}}^{i, m_{1}, 4 n-1} \cos \left(m_{1} \phi_{\alpha}+\left(4 n-1-m_{1}\right) \phi_{\beta}\right)\right)\right]\end{array}$ \\
\hline
\end{tabular}

$\dagger$ Including $(a+a),(b+b)$, and $(a+b)$.

$\hat{\sigma}_{v} \hat{H} \hat{\sigma}_{v}^{-1}=\hat{H}$ requires $\hat{\sigma}_{v} H_{+\Gamma_{k}}=(-1)^{\delta_{k 2}} H_{+\Gamma_{k}}^{*}$. Considering the real and imaginary parts of the matrix element separately, this requirement is equivalent to $\hat{\sigma}_{v} R e\left(H_{+\Gamma_{k}}\right)=$ $(-1)^{\delta_{k 2}} \operatorname{Re}\left(H_{+\Gamma_{k}}\right)$ and $\hat{\sigma}_{v} \operatorname{Im}\left(H_{+\Gamma_{k}}\right)=(-1)^{\delta_{k 1}} \operatorname{Im}\left(H_{+\Gamma_{k}}\right)$.

For the $(E+E)$-type problems,

$$
\begin{aligned}
\hat{H} & =\left(\left|+_{\alpha}\right\rangle\left\langle+_{\beta}|+|-{ }_{\beta}\right\rangle\left\langle-_{\alpha}\right|\right) H_{+_{\alpha}+_{\beta}}+\left(\left|+{ }_{\alpha}\right\rangle\left\langle-_{\beta}|+|+_{\beta}\right\rangle\left\langle-_{\alpha}\right|\right) H_{+_{\alpha}-\beta}+h c ; \\
\hat{\sigma}_{v} \hat{H} \hat{\sigma}_{v}^{-1} & =\left(\left|--_{\alpha}\right\rangle\left\langle-{ }_{\beta}|+|+{ }_{\beta}\right\rangle\left\langle+_{\alpha}\right|\right) \hat{\sigma}_{v} H_{+_{\alpha}+_{\beta}}+\left(\left|-{ }_{\alpha}\right\rangle\left\langle+_{\beta}|+|-{ }_{\beta}\right\rangle\left\langle+_{\alpha}\right|\right) \hat{\sigma}_{v} H_{+_{\alpha}-\beta}+h c .
\end{aligned}
$$

$\hat{\sigma}_{v} \hat{H} \hat{\sigma}_{v}^{-1}=\hat{H}$ requires $\left.\hat{\sigma}_{v} H_{+_{\alpha}+_{\beta}\left(+_{\alpha}-\beta\right.}\right)=H_{+_{\alpha}+_{\beta}\left(+_{\alpha}-\beta\right.}^{*}$, i.e., $\hat{\sigma}_{v} \operatorname{Re}\left(H_{+_{\alpha}+{ }_{\beta}\left(+_{\alpha}-\beta\right)}\right)=$ $\left.\operatorname{Re}\left(H_{+_{\alpha}+_{\beta}\left(+_{\alpha}-\beta\right.}\right)\right)$ and $\left.\left.\hat{\sigma}_{v} \operatorname{Im}\left(H_{+_{\alpha}+_{\beta}\left(+_{\alpha}-\beta\right.}\right)\right)=-\operatorname{Im}\left(H_{+_{\alpha}+_{\beta}\left(+_{\alpha}-\beta\right.}\right)\right)$. All in all, there are 4 additional symmetry requirements on the independent matrix elements of the 3 types of interterm problems. The requirements of the real and imaginary parts of $H_{+\Gamma_{k}}, H_{+_{\alpha}+_{\beta}}$, and $H_{+_{\alpha}-\beta}$ have a form of eigenequation. The $\hat{\sigma}_{v^{-}}$eigenvalues for the real and imaginary parts $\left(\chi_{R e}^{\sigma_{v}} \mathrm{~s}\right.$ and $\left.\chi_{I m}^{\sigma_{v}} \mathrm{~s}\right)$ of the matrix elements are summarized in Table 1. For a real $H_{i j}^{r}, \chi_{I m}^{\sigma_{v}}$ is set to zero. The two types of intraterm problems are treated similarly. For the $\Gamma_{k}$-type 
problems, $H_{\Gamma_{k} \Gamma_{k}}^{r}$ has $\chi_{R e}^{\sigma_{v}}=1$, and so does $H_{++}^{r}$ in the $E$-type problems. $H_{+-}$shares the same $\left(\chi_{R e}^{\sigma_{v}}, \chi_{I m}^{\sigma_{v}}\right)$ with $H_{+_{\alpha}-\beta}$.

Given the formulas in Tables 2 and 3 , we can use the $\hat{\sigma}_{v}$-odd and -even projection operators, $\hat{P}_{\sigma_{v}, o}=\hat{E}-\hat{\sigma}_{v}$ and $\hat{P}_{\sigma_{v}, e}=\hat{E}+\hat{\sigma}_{v}$, to construct all the expansions with $\left(\chi^{C_{4}},\left(\chi_{R e}^{\sigma_{v}}, \chi_{I m}^{\sigma_{v}}\right)\right)=( \pm 1,( \pm 1, \pm 1))$. Here, $\hat{E}$ is the identity operator, and the sign selections in the three \pm symbols are uncorrelated. One example is given here. The complete $f^{(-1)}$ expansion of the $(e+b)$ vibrational part in $C_{4}$ symmetry (Eq. 14) consists of $B_{1}$ and $B_{2}$ contributions:

$$
f^{(-1)}=f_{B 1}^{(-1)}+f_{B 2}^{(-1)}=\operatorname{Re}\left(f_{B 1}^{(-1)}\right)+i \operatorname{Im}\left(f_{B 1}^{(-1)}\right)+\operatorname{Re}\left(f_{B 2}^{(-1)}\right)+i \operatorname{Im}\left(f_{B 2}^{(-1)}\right) .
$$

Acting $\hat{P}_{\sigma_{v}, e}$ and $\hat{P}_{\sigma_{v}, o}$ on the real and imaginary parts of $f^{(-1)}$ will extract the respective $B_{1}$ and $B_{2}$ contributions, giving an $(e+b)$ expansion with $\left(\chi^{C_{4}},\left(\chi_{R e}^{\sigma_{v}}, \chi_{I m}^{\sigma_{v}}\right)\right)=(-1,(1,-1))$. Again, the resultant expansion is complete because the original $f^{(-1)}$ is complete and the projection operators fully retain the respective even and odd components. A more specific example is given for the $\left(e+b_{2}\right)$ expansion in ESI (Eqs. S4 to S7). The action of the projection operators results in constraints on the summation indices for formulas in Tables 2 and 3 . These constraints are summarized in Tables 5 and 6 . They are minimum constraints, in correspondence with the completeness of the formulas.

When $\delta_{k 1}=1, \delta_{k 2}$ must be 0 , and vice versa. Therefore, the Hamiltonian matrix elements with $\chi^{C_{4}}= \pm i$ in Table 1 have $\left(\chi_{R e}^{\sigma_{v}}, \chi_{I m}^{\sigma_{v}}\right)=( \pm 1, \mp 1)$ in pairs. The requirement of having the opposite signs in $\chi_{R e}^{\sigma_{v}}$ and $\chi_{I m}^{\sigma_{v}}$ is satisfied by all three expansions in Table 4. Each of those expansions has a phase factor of the polar angle(s) of $e$ coordinate(s), e.g., $e^{i\left(m_{1} \phi_{\alpha}+\left(4 n-1-m_{1}\right) \phi_{\beta}\right)}$ for the $(e+e)$ formula. The phase factors are all eigenstates of the angular momentum operator $\hat{L}_{z}$ ( $z$ being the $C_{4}$ axis) with eigenvalues $4 n-1$ or $2 n-1$. It is well known that the action of $\hat{\sigma}_{v}$ reverses their eigenvalues and converts them to their complex conjugates, i.e., they all have $\left(\chi_{R e}^{\sigma_{v}}, \chi_{I m}^{\sigma_{v}}\right)=(1,-1)$. In the $(e+v)$ expansions, the phase factors are multiplied by monomials of $v_{k}^{M}$, which flip their signs under $\hat{\sigma}_{v}$ when $k=2$ and $M$ is odd. Such a sign-flipping gives $\left(\chi_{R e}^{\sigma_{v}}, \chi_{I m}^{\sigma_{v}}\right)=(-1,1)$ for the products. Now it is clear that all monomials in the expansions in Table 4 satisfy $\left(\chi_{R e}^{\sigma_{v}}, \chi_{I m}^{\sigma_{v}}\right)=( \pm 1, \mp 1)$ in pairs. If a monomial is multiplied by a real coefficient, its $\left(\chi_{R e}^{\sigma_{v}}, \chi_{I m}^{\sigma_{v}}\right)$ is invariant. If it is multiplied by a purely imaginary coefficient, $\chi_{R e}^{\sigma_{v}}$ and $\chi_{I m}^{\sigma_{v}}$ are swapped. Therefore, to have $\hat{\sigma}_{v}$-adapted expansions from Table 4 , the coefficients there must be either real or purely 
TABLE 5. Constraints on expansions in Table 2 to give the appropriate $\chi_{R e}^{\sigma_{v}}$ and $\chi_{I m}^{\sigma_{v}}$. When $\chi_{I m}^{\sigma_{v}}=0$, only the real part of the corresponding entry in Table 2 should be considered.

\begin{tabular}{|c|c|c|c|}
\hline Modes & $(1,(1,0))$ & $(1,(-1,0))$ & $(1,(1,-1))$ \\
\hline$\left(a_{1}+a_{1}\right)$ & $\mathrm{nr}^{\dagger}$ & na & na \\
\hline$\left(a_{1}+a_{2}\right)^{\ddagger}$ & $I_{2}$ even & $I_{2}$ odd & $I_{2}$ even, $I_{4}$ odd \\
\hline$\left(a_{2}+a_{2}\right)$ & $I_{1}, I_{2}$ ee or oo ${ }^{\S}$ & $I_{1}, I_{2}$ eo or oe e $^{£}$ & $\begin{array}{l}I_{1}, I_{2} \text { ee or oo, } \\
I_{3}, I_{4} \text { eo or oe }\end{array}$ \\
\hline$\left(a_{1}+b_{1}\right),\left(a_{1}+b_{2}\right)$ & $\mathrm{nr}$ & na & na \\
\hline$\left(a_{2}+b_{1}\right),\left(a_{2}+b_{2}\right)$, & $I_{1}$ even & $I_{1}$ odd & $I_{1}$ even, $I_{2}$ odd \\
\hline$\left(b_{1}+b_{1}\right),\left(b_{2}+b_{2}\right)$ & $\mathrm{nr}$ & na & na \\
\hline$\left(b_{1}+b_{2}\right)$ & $a_{e e} \mathrm{nz}^{\$}$ & $a_{o o} \mathrm{nz}^{\$}$ & $a_{e e}^{r}, a_{o o}^{i} \mathrm{nz}$ \\
\hline$\left(e+a_{1}\right),\left(e+b_{1}\right)$ & $\cos n z^{\#}$ & $\sin n z$ & $a^{r} \mathrm{nz}$ \\
\hline$\left(e+a_{2}\right)$ & $I_{1}$ even, $I_{2}$ odd & $I_{1}$ odd, $I_{2}$ even & $I_{1}$ even, $I_{2}$ odd \\
\hline$\left(e+b_{2}\right)$ & $\begin{array}{l}\cos \mathrm{nz} \text { if } m \text { even, } \\
\sin \mathrm{nz} \text { if } m \text { odd }\end{array}$ & $\begin{array}{l}\sin \mathrm{nz} \text { if } m \text { even, } \\
\cos \mathrm{nz} \text { if } m \text { odd }\end{array}$ & $\begin{array}{c}a^{r} \mathrm{nz} \text { if } m \text { even, } \\
a^{i} \mathrm{nz} \text { if } m \text { odd }\end{array}$ \\
\hline$(e+e)$ & $\cos n z$ & $\sin n z$ & $a^{r} \mathrm{nz}$ \\
\hline
\end{tabular}

$\dagger$ "nr" means "no restriction". ${ }^{\ddagger}$ For two modes whose irreps only differ in subscripts, $\alpha$-subscripted coordinates in Table 2 are for the first ( $a_{1}$ here) and $\beta$-for the second ( $a_{2}$ here) mode. $I_{2}$ needs to be even. ${ }^{\S} I_{1}$ and $I_{2}$ need to be both even or both odd. ${ }^{£}$ When $I_{1}$ is even, $I_{2}$ must be odd, and vice versa. ${ }^{\$}$ $a_{e e}$ means $a_{2 J_{1}, 2 J_{2}} . a_{o o}$ means $a_{2 J_{1}+1,2 J_{2}+1}$. "nz" means only these coefficients are nonzero. \# Only the terms associated with cos factors are nonzero.

imaginary. The real/imaginary selections are translated to constraints that are summarized in Table 7. Expansion formulas with $\left(\chi^{C_{4}}=-i,\left(\chi_{R e}^{\sigma_{v}}, \chi_{I m}^{\sigma_{v}}\right)\right)$ are obtained by taking complex conjugates of the counterparts with $\left(\chi^{C_{4}}=i,\left(\chi_{R e}^{\sigma_{v}}, \chi_{I m}^{\sigma_{v}}\right)\right)$. These constraints do not abandon any monomials in the complete expansions in Table 4. Only the necessary selections of real and imaginary coefficients are taken. Therefore, these are minimum constraints and the resultant $\hat{\sigma}_{v}$-eigen-expansions are complete.

With all the 7 tables, expansion formulas for any of the 225 (15 electronic $\times 15$ vibrational 
TABLE 6. Constraints on expansions in Table 3 to give the appropriate $\chi_{R e}^{\sigma_{v}}$ and $\chi_{I m}^{\sigma_{v}}$. When $\chi_{I m}^{\sigma_{v}}=0$, only the real part of the corresponding entry in Table 3 should be considered.

\begin{tabular}{|c|c|c|c|}
\hline Modes & $(-1,(1,0))$ & $(-1,(-1,0))$ & $(-1,(1,-1))$ \\
\hline$\left(a_{1}+b_{1}\right)$ & $\mathrm{nr}$ & na & na \\
\hline$\left(a_{1}+b_{2}\right)$ & na & $\mathrm{nr}$ & na \\
\hline$\left(a_{2}+b_{1}\right)$ & $I_{1}$ even & $I_{1}$ odd & $I_{1}$ even, $I_{2}$ odd \\
\hline$\left(a_{2}+b_{2}\right)$ & $I_{1}$ odd & $I_{1}$ even & $I_{1}$ odd, $I_{2}$ even \\
\hline$\left(b_{1}+b_{1}\right)$ & $\mathrm{nr}$ & na & na \\
\hline$\left(b_{1}+b_{2}\right)$ & $b_{o e} \mathrm{nz}$ & $b_{e o} \mathrm{nz}$ & $b_{o e}^{r}, b_{e o}^{i} \mathrm{nz}$ \\
\hline$\left(b_{2}+b_{2}\right)$ & na & $\mathrm{nr}$ & na \\
\hline$\left(e+a_{1}\right),\left(e+b_{1}\right)$ & $\cos n z$ & $\sin n z$ & $b^{r} \mathrm{nz}$ \\
\hline$\left(e+a_{2}\right)$ & $I_{1}$ even, $I_{2}$ odd & $I_{1}$ odd, $I_{2}$ even & $I_{1}$ even, $I_{2}$ odd \\
\hline$\left(e+b_{2}\right)$ & $\begin{array}{l}\cos n z \text { if } m \text { odd, } \\
\sin n z \text { if } m \text { even }\end{array}$ & $\begin{array}{l}\sin \mathrm{nz} \text { if } m \text { odd, } \\
\cos \mathrm{nz} \text { if } m \text { even }\end{array}$ & $\begin{array}{l}b^{r} \mathrm{nz} \text { if } m \text { odd, } \\
b^{i} \mathrm{nz} \text { if } m \text { even }\end{array}$ \\
\hline$(e+e)$ & $\cos n z$ & $\sin n z$ & $b^{r} \mathrm{nz}$ \\
\hline
\end{tabular}

TABLE 7. Constraints on expansions in Table 4 to give the appropriate $\chi_{R e}^{\sigma_{v}}$ and $\chi_{I m}^{\sigma_{v}}$.

\begin{tabular}{ccc}
\hline Modes & $(i,(1,-1))$ & $(i,(-1,1))$ \\
\hline$\left(e+a_{1}\right),\left(e+b_{1}\right)$ & $c^{r} \mathrm{nz}$ & $c^{i} \mathrm{nz}$ \\
\hline$\left(e+a_{2}\right)$ & $I_{1}$ even, $I_{2}$ odd & $I_{1}$ odd, $I_{2}$ even \\
\hline$\left(e+b_{2}\right)$ & $c^{r}\left(c^{i}\right) \mathrm{nz}$ if $n$ even $($ odd $)$ & $c^{r}\left(c^{i}\right) \mathrm{nz}$ if $n$ odd (even) \\
\hline$(e+e)$ & $c^{r} \mathrm{nz}$ & $c^{i} \mathrm{nz}$ \\
\hline
\end{tabular}

parts $)$ interterm and $75(5 \times 15)$ intraterm vibronic problems in $C_{4 v}$ symmetry are readily obtained. The procedure of obtaining the $\left(E+A_{2} / B_{2}\right) \otimes\left(e+b_{2}\right)$ expansion formulas is demonstrated here as examples. We first look for $\left(\chi^{C_{4}},\left(\chi_{R e}^{\sigma_{v}}, \chi_{I m}^{\sigma_{v}}\right)\right)$ for the matrix elements of the electronic part in Table $1,(i,(-1,1))$ for $H_{+A_{2}}$ in the $\left(E+A_{2}\right) \otimes\left(e+b_{2}\right)$ problem. $\chi^{C_{4}}=i$ guides us to Table 4 to look for the expansion formula for the vibrational part, the 
$(e+b)$ row in this case:

$$
\begin{aligned}
H_{+A}= & w^{\bmod (n, 2)+2 I} \rho^{|2 n-1|+2 K}\left[c_{2 I, 2 K}^{r, 2 n-1} \cos (2 n-1) \phi-c_{2 I, 2 K}^{i, 2 n-1} \sin (2 n-1) \phi\right. \\
& \left.+i c_{2 I, 2 K}^{r, 2 n-1} \sin (2 n-1) \phi+i c_{2 I, 2 K}^{i, 2 n-1} \cos (2 n-1) \phi\right] .
\end{aligned}
$$

Finally, $\left(\chi^{C_{4}},\left(\chi_{R e}^{\sigma_{v}}, \chi_{I m}^{\sigma_{v}}\right)\right)$ guide us to the $\left(e+b_{2}\right)-(i,(-1,1))$ entry in Table 7 . With the constraints of " $c^{r}\left(c^{i}\right)$ nz if $n$ odd (even)", Eq. 21 becomes

$$
H_{+A_{2}}=c_{2 I, 2 K}^{r, 4 k+1} w^{2 I+1} \rho^{|4 k+1|+2 K} e^{i(4 k+1) \phi}+i c_{2 I, 2 K}^{i, 4 k-1} w^{2 I} \rho^{|4 k-1|+2 K} e^{i(4 k-1) \phi},
$$

where $2 k$ and $2 k+1$ are introduced to differentiate even and odd $n$. Since $H_{+A_{2}}$ and $H_{+B_{2}}$ only differ in $\chi^{C_{4}}(i$ vs. $-i$, see Table 1$)$, the $H_{+B_{2}}$ expansion in the $\left(E+B_{2}\right) \otimes\left(e+b_{2}\right)$ problem is the complex conjugate of Eq. 22:

$$
H_{+B_{2}}=c_{2 I, 2 K}^{r, 4 k-1} w^{2 I+1} \rho^{|4 k-1|+2 K} e^{i(4 k-1) \phi}-i c_{2 I, 2 K}^{i, 4 k+1} w^{2 I} \rho^{|4 k+1|+2 K} e^{i(4 k+1) \phi} .
$$

Transforming into the real $|X\rangle$ and $|Y\rangle$ states and cartesian $x$ and $y$ coordinates, and keeping up to second order terms, we have

$$
H_{X B_{2}}=\sqrt{2}\left(c_{0,0}^{i, 1} y+c_{0,0}^{r,-1} w x\right) ; H_{Y B_{2}}=\sqrt{2}\left(c_{0,0}^{i, 1} x+c_{0,0}^{r,-1} w y\right) .
$$

They agree with the $\left(E+B_{2}\right) \otimes\left(e+b_{2}\right)$ block in Eq. 31 in Ref. 32 .

All the $C_{4 v}$ formulas are applicable to (p)JT problems in $D_{4}$ and $D_{2 d}$ symmetries, as long as the $E$ and $e$ components transform as in Eq. 16, but with $\sigma_{v}$ being replaced by $C_{2}^{\prime}$ in the respective symmetries (e.g., Figure $1(\mathrm{~d})$ and (e)).

\section{C. $C_{4 h}$ Problems.}

The inversion center is the additional symmetry element that raises $C_{4}$ symmetry to $C_{4 h}$ symmetry. In $C_{4 h}$ symmetry, there is no additional requirement on the $E$ component states and $e$ component modes other than satisfying Eq. 2. The $E$ and $e$ components in this symmetry shall hence have similar orientations as in Figure 1(a). All states and vibrational modes are eigenstates of the inversion operator $\hat{I}$, with eigenvalues 1 or -1 determined by their parities $g$ or $u$. In polar coordinates of an $e$ mode, the parity is associated to $\rho$, i.e.,

$$
\hat{I} f\left(v_{g}, v_{u}, \rho_{\alpha, g}, \phi_{\alpha}, \rho_{\beta, u}, \phi_{\beta}\right)=f\left(v_{g},-v_{u}, \rho_{\alpha, g}, \phi_{\alpha},-\rho_{\beta, u}, \phi_{\beta}\right) .
$$


To have an $\hat{I}$-invariant Hamiltonian, each matrix element $H_{i j}$ must have a parity that is the product of the parities of states $i$ and $j$, i.e., the $(-1)^{\delta_{p q}+1} \hat{I}$-eigenvalues $\left(\chi^{I} \mathrm{~s}\right)$ in Table 1 . Henceforth, the $g / u$ subscripts are labelled by $p$ and $q$. The only constraint on the root formulas in Table 2 to 4 to obtain expansions of the $C_{4 h}$ problems is that the total powers of $u$ coordinates needs to be even (odd) for $H_{i j}$ s with $\chi^{I}=1(-1)$. This simple rule is translated into constraints that are summarized in Tables S1 to S3 in ESI. These constraints are obtained by applying the $\hat{I}$-odd $(\hat{E}-\hat{I})$ and -even $(\hat{E}+\hat{I})$ projection operators onto the root formulas in Tables 2 to 4 . The completeness of the root formulas and the fact that the projection operators fully retain the components with the respective parities ensure the completeness of the resultant expansions and the minimum nature of the constraints. Expansion formulas with $\left(\chi^{C_{4}}=-i, \chi^{I}\right)$ are obtained by taking complex conjugates of those with $\left(\chi^{C_{4}}=i, \chi^{I}\right)$. The tabulated root formulas and constraints readily give Hamiltonian expansions for all 441 (21 electronic $\times 21$ vibronic parts) interterm and $126(6 \times 21)$ intraterm problems in $C_{4 h}$ symmetry.

\section{D. $D_{4 h}$ Problems.}

The $D_{4 h}$ symmetry is a composite of the $C_{4 h}$ and the $D_{4}$ (or $D_{2 d}$ ) symmetries. We do not view it as a composite of the $C_{4 h}$ and the $C_{4 v}$ symmetries, although we could. This is because in the $D_{4 h}$ character table, the subscripts 1 and 2 of $\Gamma$ indicate being $\hat{C}_{2}^{\prime}$-even and -odd, instead of $\hat{\sigma}_{v^{-}}$. Therefore, in the $D_{4 h}$ derivation, the $E$ and $e$ components transform as in Eq. 16 but with $\hat{\sigma}_{v}$ being replaced by $\hat{C}_{2}^{\prime}$ (e.g., Figure $\left.1(\mathrm{f})\right)$. In this composite symmetry, each of the matrix elements $H_{i j}$ needs to have all four eigenvalues of the three symmetry operators: $\left(\chi^{C_{4}},\left(\chi_{R e}^{C_{2}^{\prime}}, \chi_{I m}^{C_{2}^{\prime}}\right), \chi^{I}\right)$. Note that $\left(\chi_{R e}^{\sigma_{v}}, \chi_{I m}^{\sigma_{v}}\right)$ have been replaced by $\left(\chi_{R e}^{C_{2}^{\prime}}, \chi_{I m}^{C_{2}^{\prime}}\right)$. The expansion for $H_{i j}$ with $\left(\chi^{C_{4}},\left(\chi_{R e}^{C_{2}^{\prime}}, \chi_{I m}^{C_{2}^{\prime}}\right), \chi^{I}\right)$ simply adopts the corresponding $\chi^{C_{4}}$ root formula with the combined $\left(\chi^{C_{4}},\left(\chi_{R e}^{C_{2}^{\prime}}, \chi_{\text {Im }}^{C_{2}^{\prime}}\right)\right)$ and $\left(\chi^{C_{4}}, \chi^{I}\right)$ constraints. The completeness of the resultant formula is guaranteed by the completeness of the root formula and the minimum nature of each set of the constraints.

Here we use the $\left(E_{g}+A_{1 u}\right) \otimes\left(e_{g}+b_{2 u}\right)$ problem as an example to show how to obtain Hamiltonian expansion in $D_{4 h}$ symmetry. The $\left(E_{g}+A_{1 u}\right) \otimes\left(e_{g}+b_{2 u}\right)$ problem is decomposed into the $\left(E+A_{1}\right) \otimes\left(e+b_{2}\right)$ problem in $D_{4}$ symmetry and the $\left(E_{g}+A_{u}\right) \otimes\left(e_{g}+b_{u}\right)$ problem in $C_{4 h}$ symmetry. There is one matrix element $H_{+A}$ for the $(E+A)$ electronic part. From 
the $(E+A)$ blocks in Tables 1 , we know its $\left(\chi^{C_{4}},\left(\chi_{R e}^{C_{2}^{\prime}}, \chi_{I m}^{C_{2}^{\prime}}\right), \chi^{I}\right)=(i,(1,-1),-1)$. We first get the $(E+A) \otimes(e+b)$ root expansion formula from Table 4:

$$
H_{+A}=w^{\bmod (n, 2)+2 I} \rho^{|2 n-1|+2 K}\left[c_{2 I, 2 K}^{r, 2 n-1}+i c_{2 I, 2 K}^{i, 2 n-1}\right] e^{i(2 n-1) \phi} .
$$

Imposing the $(i,(1,-1))$ constraints of " $c^{r}\left(c^{i}\right)$ nz if $n$ even (odd)" for $\left(e+b_{2}\right)$ in Table 7 , the formula becomes

$$
H_{+A_{1}}=c_{2 I, 2 K}^{r, 4 k-1} w^{2 I} \rho^{|4 k-1|+2 K} e^{i(4 k-1) \phi}+i c_{2 I, 2 K}^{i, 4 k+1} w^{2 I+1} \rho^{|4 k+1|+2 K} e^{i(4 k+1) \phi},
$$

where even and odd $n$ s have been represented by $2 k$ and $2 k+1$, corresponding to the first and the second terms. The $\left(e_{g}+b_{u}\right)-(i,-1)$ entry in Table S3 is "n odd". Therefore, only the second term remains and

$$
H_{+g} A_{1 u}=i c_{2 I, 2 K}^{i, 4 k+1} w^{2 I+1} \rho^{|4 k+1|+2 K} e^{i(4 k+1) \phi} .
$$

This is the expansion formula for the $\left(E_{g}+A_{1 u}\right) \otimes\left(e_{g}+b_{2 u}\right)$ problem. Through similar procedures, we can readily obtain vibronic Hamiltonians for all 3025 (55 electronic $\times 55$ vibrational parts) interterm and $550(10 \times 55)$ intraterm problems in $D_{4 h}$ symmetry.

Numerical Tests. The textbook problem of $E \otimes\left(b_{1}+b_{2}\right)$ in $D_{2 d}$ symmetry is taken as the first example to compare our expansion formulas with numerical calculations. The $E$ entries in Table 1 , the $(b+b)$ formulas in Tables 2 and 3 , and the $\left(b_{1}+b_{2}\right)$ constraints corresponding to the appropriate eigenvalues of symmetry operators in Tables 5 and 6 give:

$$
H_{++}=a_{2 J_{1}, 2 J_{2}}^{r} w_{1}^{2 J_{1}} w_{2}^{2 J_{2}} ; H_{+-}=b_{2 J_{1}+1,2 J_{2}}^{r} w_{1}^{2 J_{1}+1} w_{2}^{2 J_{2}}+i b_{2 J_{1}, 2 J_{2}+1}^{i} w_{1}^{2 J_{1}} w_{2}^{2 J_{2}+1}
$$

$w_{1 / 2}$ is the $b_{1 / 2}$ coordinate. Transforming to the real $|X\rangle$ and $|Y\rangle$ states (Eq. 3), we have

$$
\frac{H_{X X}+H_{Y Y}}{2}=H_{++} ; \frac{H_{X X}-H_{Y Y}}{2}=b_{2 J_{1}+1,2 J_{2}}^{r} w_{1}^{2 J_{1}+1} w_{2}^{2 J 2} ; H_{X Y}=-b_{2 J_{1}, 2 J_{2}+1}^{i} w_{1}^{2 J_{1}} w_{2}^{2 J_{2}+1} \text {. }
$$

A triplet ethylene model is used to examine these three expansion formulas. The $D_{2 d}$ twisted triplet ethylene structure optimized at the unrestricted B3LYP ${ }^{33,34}$ level with the aug-ccpVTZ basis set ${ }^{35}$ is taken as the reference structure. All calculations are performed using GAMESS-US. ${ }^{36}$ The one-electron model Hamiltonian operator contains kinetic and nucleielectron attraction operators. The delocalized $\pi$ molecular orbitals (MOs) that correlate with the HOMO and LUMO of ground state ethylene are taken as the one-electron $|X\rangle$ and $|Y\rangle$ 
states. The ethylene is distorted along the $b_{1}$ torsional and the $b_{2}$ scissoring normal modes. The orbitals and modes are displayed in Figure 3. The orbitals are frozen with respect to the distortion, i.e., they are genuine one-electron diabatic states. No diabatization is needed.

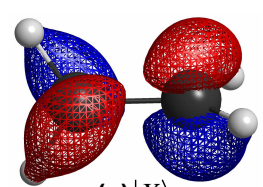

(a) $|X\rangle$

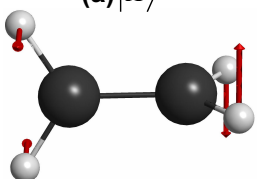

(c) $b_{1}$ mode, $720 \mathrm{~cm}^{-1}$

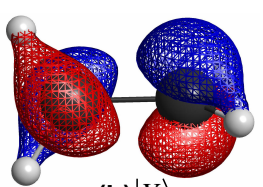

(b) $|Y\rangle$

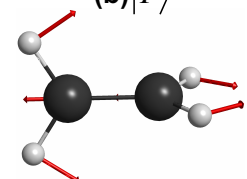

(d) $b_{2}$ mode, $1425 \mathrm{~cm}^{-1}$

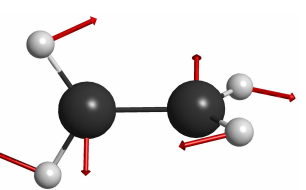

(e) $e_{x}$ mode, $936 \mathrm{~cm}^{-1}$ (f) $e_{y}$ mode, $936 \mathrm{~cm}^{-1}$

FIG. 3. The orbitals and vibrational modes of the triplet ethylene used in the numerical tests of the $E \otimes\left(b_{1}+b_{2}\right)$ and $E \otimes e$ problems in $D_{2 d}$ symmetry.

The Hamiltonian matrix elements are calculated on a square mesh of $w_{1}$ and $w_{2}$ from -1 to $1 \sqrt{u} \AA$ in both dimensions, with a $0.1 \sqrt{u} \AA$ interval. They are combined as the left hand sides in Eq. 30 and fitted against free polynomials with all $44 w_{1}^{l} w_{2}^{m}$ monomials up to eighth order. The resultant expansions in the unit of $\mathrm{eV}$ are:

$$
\begin{aligned}
\frac{H_{X X}+H_{Y Y}}{2}= & 2.57 w_{1}^{2}+4.21 w_{2}^{2}-1.74 w_{1}^{4}-2.68 w_{1}^{2} w_{2}^{2}-2.11 w_{2}^{4}+1.56 w_{1}^{6}+1.87 w_{1}^{4} w_{2}^{2}+1.90 w_{1}^{2} w_{2}^{4} \\
& +1.53 w_{2}^{6}-0.58 w_{1}^{8}-0.56 w_{1}^{6} w_{2}^{2}-0.62 w_{1}^{4} w_{2}^{4}-0.55 w_{1}^{2} w_{2}^{6}-0.54 w_{2}^{8} \\
\frac{H_{X X}-H_{Y Y}}{2}= & 5.57 w_{1}-3.16 w_{1}^{3}-3.07 w_{1} w_{2}^{2}+1.27 w_{1}^{5}+2.06 w_{1}^{3} w_{2}^{2}+0.94 w_{1} w_{2}^{4}-0.28 w_{1}^{7} \\
& -0.54 w_{1}^{5} w_{2}^{2}-0.45 w_{1}^{3} w_{2}^{4}-0.13 w_{1} w_{2}^{6} \\
H_{X Y}= & -0.15 w_{2}+0.07 w_{1}^{2} w_{2}-0.06 w_{1}^{4} w_{2}-0.08 w_{1}^{2} w_{2}^{3}-0.02 w_{2}^{5}+0.01 w_{1}^{6} w_{2} \\
& +0.02 w_{1}^{4} w_{2}^{3}+0.02 w_{1}^{2} w_{2}^{5}+0.005 w_{2}^{7}
\end{aligned}
$$

All the other terms have too small coefficients to consider. The $\frac{H_{X X}+H_{Y Y}}{2}$ value at $w_{1}=$ $w_{2}=0$ has been set to 0 . The fitting automatically annihilates the terms that are nullified by symmetry, and the fitted results are in perfect agreement with the expansions in Eq. 30.

The second example of the $E \otimes e$ problem in $D_{2 d}$ symmetry demonstrates how to obtain formulas for unimodal problems. This is a subproblem of any of the $E \otimes\left(e+\gamma_{k}\right)$ and 
$E \otimes(e+e)$ bimodal problems. We arbitrarily choose to start with the $E \otimes\left(e+b_{1}\right)$ formulas in deriving the $E \otimes e$ formulas. From the $E$ entries in Table 1, the $(e+b)$ formulas in Tables 2 and 3 , and the $\left(e+b_{1}\right)$ constraints corresponding to the appropriate $\hat{C}_{2}^{\prime}$-eigenvalues $\left(=\hat{\sigma}_{v}\right.$-eigenvalues $)$ in Tables 5 and 6 , we obtain the expansions:

$$
H_{++}=a_{2 I, 2 K}^{r, 2 m} w^{\bmod (m, 2)+2 I} \rho^{2 m+2 K} \cos 2 m \phi ; H_{+-}=b_{2 I, 2 K}^{r, 2 m} w^{\bmod (m, 2)+2 I+1} \rho^{|2 m|+2 K} e^{i 2 m \phi} .
$$

Note that $|2 m|$ has been replaced by $2 m$ in $H_{++}$because of the evenness of $\cos 2 m \phi$; this $m$ only takes nonnegative integers. The $E \otimes e$ formulas are obtained by setting $w=0$; only the terms with a zero power of $w$ remain, i.e., $m=2 k$ and $2 I=0$ in $H_{++}$and $m=2 k+1$ and $2 I=-2$ in $H_{+-}$:

$$
H_{++}=a_{0,2 K}^{r, 4 k} \rho^{4 k+2 K} \cos 4 k \phi ; H_{+-}=b_{-2,2 K}^{r, 4 k+2} \rho^{|4 k+2|+2 K} e^{i(4 k+2) \phi} .
$$

Transforming to the real $E$ components,

$\frac{H_{X X}+H_{Y Y}}{2}=H_{++} ; \frac{H_{X X}-H_{Y Y}}{2}=b_{-2,2 K}^{r, 4 k+2} \rho^{|4 k+2|+2 K} \cos (4 k+2) \phi ; H_{X Y}=-b_{-2,2 K}^{r, 4 k+2} \rho^{|4 k+2|+2 K} \sin (4 k+2) \phi$.

Keeping up to sixth order terms,

$$
\begin{aligned}
\frac{H_{X X}+H_{Y Y}}{2}= & a_{0,2}^{r, 0} \rho^{2}+a_{0,4}^{r, 0} \rho^{4}+a_{0,0}^{r, 4} \rho^{4} \cos 4 \phi+a_{0,0}^{r, 0} \rho^{6}+a_{0,2}^{r, 4} \rho^{6} \cos 4 \phi \\
= & a_{0,2}^{r, 0}\left(x^{2}+y^{2}\right)+\left(a_{0,4}^{r, 0}+a_{0,0}^{r, 4}\right)\left(x^{4}+y^{4}\right)+\left(2 a_{0,4}^{r, 0}-6 a_{0,0}^{r, 4}\right) x^{2} y^{2}+\left(a_{0,6}^{r, 0}+a_{0,2}^{r, 4}\right)\left(x^{6}+y^{6}\right) \\
& +\left(4 a_{0,6}^{r, 0}-6 a_{0,2}^{r, 4}\right) x^{2} y^{2}\left(x^{2}+y^{2}\right) ; \\
\frac{H_{X X}-H_{Y Y}}{2}= & \left(b_{-2,0}^{r, 2}+b_{-2,0}^{r,-2}\right) \rho^{2} \cos 2 \phi+\left(b_{-2,2}^{r, 2}+b_{-2,2}^{r,-2}\right) \rho^{4} \cos 2 \phi+\left(b_{-2,4}^{r,-2}+b_{-2,4}^{r, 2}\right) \rho^{6} \cos 2 \phi \\
& +\left(b_{-2,0}^{r,-6}+b_{-2,0}^{r, 6}\right) \rho^{6} \cos 6 \phi \\
= & \left(b_{-2,0}^{r, 2}+b_{-2,0}^{r,-2}\right)\left(x^{2}-y^{2}\right)+\left(b_{-2,2}^{r, 2}+b_{-2,2}^{r,-2}\right)\left(x^{4}-y^{4}\right)+\left(b_{-2,4}^{r,-2}+b_{-2,4}^{r, 2}+b_{-2,0}^{r,-6}+b_{-2,0}^{r, 6}\right)\left(x^{6}-y^{6}\right) \\
& +\left(b_{-2,4}^{r,-2}+b_{-2,4}^{r, 2}+15 b_{-2,0}^{r,-6}+15 b_{-2,0}^{r, 6}\right) x^{2} y^{2}\left(x^{2}-y^{2}\right) ; \\
= & \left(b_{-2,0}^{r,-2}-b_{-2,0}^{r, 2}\right) \rho^{2} \sin 2 \phi+\left(b_{-2,2}^{r,-2}-b_{-2,2}^{r, 2}\right) \rho^{4} \sin 2 \phi+\left(b_{-2,4}^{r,-2}-b_{-2,4}^{r, 2}\right) \rho^{6} \sin 2 \phi \\
& +\left(b_{-2,0}^{r,-6}-b_{-2,0}^{r, 6}\right) \rho^{6} \sin 6 \phi \\
= & \left(b_{-2,0}^{r,-2}-b_{-2,0}^{r, 2}\right) 2 x y+\left(b_{-2,2}^{r,-2}-b_{-2,2}^{r, 2}\right) 2 x y\left(x^{2}+y^{2}\right)+\left(b_{-2,4}^{r,-2}-b_{-2,4}^{r, 2}+3 b_{-2,0}^{r,-6}-3 b_{-2,0}^{r, 6}\right) 2 x y\left(x^{4}+y^{4}\right) \\
& +\left(4 b_{-2,4}^{r,-2}-4 b_{-2,4}^{r, 2}-20 b_{-2,0}^{r,-6}+20 b_{-2,0}^{r, 6}\right) x^{3} y^{3} .
\end{aligned}
$$

The second order terms in all three expansions are consistent with the second order $E \otimes e$ block in Eq. 23 in Ref. 32. The $(4 k+2) \phi$ angular factor in Eq. 34 determines that the 
lowest order $E \otimes e$ coupling is at the second order, which is similar (but not identical) to the $\Pi \otimes \pi$ Renner-Teller coupling in linear molecules. ${ }^{7}$ Using the $E$ component states and the $e$ component rocking modes shown in Figure 3, we calculate those matrix elements on a $x-y$ square mesh similar to the $w_{1}-w_{2}$ mesh above and fit the data against free polynomials with all $27 x^{l} y^{m}$ monomials up to sixth order. The resultant expansions of the matrix elements in $\mathrm{eV}$ are:

$$
\begin{aligned}
\frac{H_{X X}+H_{Y Y}}{2} & =2.45\left(x^{2}+y^{2}\right)-0.47\left(x^{4}+y^{4}\right)-2.93 x^{2} y^{2}+0.12\left(x^{6}+y^{6}\right)+0.78 x^{2} y^{2}\left(x^{2}+y^{2}\right) \\
\frac{H_{X X}-H_{Y Y}}{2} & =-1.81\left(x^{2}-y^{2}\right)+0.31\left(x^{4}-y^{4}\right)-0.04\left(x^{6}-y^{6}\right)-0.07 x^{2} y^{2}\left(x^{2}-y^{2}\right) \\
H_{X Y} & =4.39 x y+0.59 x y\left(x^{2}+y^{2}\right)-0.04 x y\left(x^{4}+y^{4}\right)-0.82 x^{3} y^{3}
\end{aligned}
$$

The numerical results are again in perfect agreement with the derived formulas. The two tests raise our confidence in the robustness of our derivation.

Generalization of the derivation to the other axial symmetries. The derivation here is applicable to (p)JT problems in all axial symmetries, i.e., all the $C_{n}, S_{n}, C_{n v}, C_{n h}$, $D_{n}, D_{n d}$, and $D_{n h}$ symmetries. It is well known that in a $C_{n}$ symmetry, a set of (p)JTcoupled diabats, including all degenerate components, can always be transformed to be $\hat{C}_{n}$-eigenstates, with possible eigenvalues

$$
e^{i M 2 \pi / n}, M=0, \pm 1, \pm 2, \cdots, \pm \operatorname{Int}(n / 2)
$$

Int means taking the integer part of the argument. $M=0$ corresponds to $A$ states. Only when $n$ being even, $M= \pm n / 2$ gives the same $\hat{C}_{n}$-eigenvalue -1 and corresponds to $B$ states. In the text below, we do not differentiate $n$ being odd or even. One should keep in mind that statements about $B$ states and mathematical expressions with $n / 2$ are applicable only when $n$ is even. $A$ and $B$ states are chosen to be real, i.e., $A$ states transform like $\sin m n \phi$ and $\cos m n \phi$, and $B$ states transform like $\sin \frac{m n}{2} \phi$ and $\cos \frac{m n}{2} \phi$, recalling that $\phi$ is the polar angle about the $C_{n}$ axis. All the other $\pm M$ pairs exist only for $n>2$ and they correspond to $E_{M}$ degenerate states. We use $M$ to label the states. $|M\rangle$ and $|-M\rangle$ are complex conjugates

of each other and are interconverted by the time-reversal operator. Note that this is also true for $A$ and $B$ states. For an $A$ state, it is obvious that $|0\rangle=|-0\rangle$. Only one of $|n / 2\rangle$ and $|-n / 2\rangle$ shall be kept. We formally associate both of them to the same real $B$ state i.e., $|n / 2\rangle=|-n / 2\rangle$ and they are real. This is only a symbolism setting that gives the compact Hamiltonian expression in Eq. 38. 
With the time-reversal symmetry being enforced, the Hamiltonian operator expanded in the set of diabats can be written in a form of

$$
\hat{H}=\left(|M\rangle\left\langle M^{\prime}|+|-M^{\prime}\right\rangle\langle-M|\right) H_{M M^{\prime}}+h c .
$$

Please note the hidden summation over all relevant states $\{|M\rangle\}$ and $\left\{\left|M^{\prime}\right\rangle\right\}$. For the $M=M^{\prime}$ diagonal terms and the terms with $M$ and $M^{\prime}=0$ or $n / 2, H_{M M^{\prime}}$ is real, and $H_{M M^{\prime}}$ of these terms takes $\frac{\left\langle M|\hat{H}| M^{\prime}\right\rangle}{2}$ to counteract the duplication of $+h c$. Furthermore, for the diagonal terms with $M=M^{\prime}=0$ or $n / 2$, the two dyads in the parentheses are identical and $H_{M M}$ takes $\frac{\langle M|\hat{H}| M\rangle}{4}$ to counteract the two-fold duplications.

$$
\hat{C}_{n} \hat{H} \hat{C}_{n}^{-1}=e^{i\left(M-M^{\prime}\right) 2 \pi / n}\left(|M\rangle\left\langle M^{\prime}|+|-M^{\prime}\right\rangle\langle-M|\right) \hat{C}_{n} H_{M M^{\prime}}+h c .
$$

$\hat{C}_{n} \hat{H} \hat{C}_{n}^{-1}=\hat{H}$ requires $\hat{C}_{n} H_{M M^{\prime}}=e^{i\left(M^{\prime}-M\right) 2 \pi / n} H_{M M^{\prime}}$, i.e., each $H_{M M^{\prime}}$ is characterized by a $\hat{C}_{n}$-eigenvalue $\left(\chi^{C_{n}}\right)$, which takes the same range of values as in Eq. 37.

In $C_{n v}$ symmetry, we can always adjust the phases of the states so that $\sigma_{v}| \pm M\rangle=$ $(-1)^{\delta_{k 2}}|\mp M\rangle$. Note that the $(-1)^{\delta_{k 2}}$ only applies when $M=0$ and $\pm n / 2$; it indicates a possible sign-flipping for $A_{k}$ and $B_{k}$ states depending on $k=1$ or 2 . Consequently,

$$
\hat{\sigma}_{v} \hat{H} \hat{\sigma}_{v}^{-1}=(-1)^{\delta_{k 2}}(-1)^{\delta_{k^{\prime} 2}}\left(|-M\rangle\left\langle-M^{\prime}|+| M^{\prime}\right\rangle\langle M|\right) \hat{\sigma}_{v} H_{M M^{\prime}}+h c
$$

$\hat{\sigma}_{v} \hat{H} \hat{\sigma}_{v}^{-1}=\hat{H}$ requires $\hat{\sigma}_{v} H_{M M^{\prime}}=(-1)^{\delta_{k 2}}(-1)^{\delta_{k^{\prime} 2}} H_{M M^{\prime}}^{*}$. Therefore, each $H_{M M^{\prime}}$ is characterized by $\hat{\sigma}_{v^{-}}$eigenvalues $\left(\chi_{R e}^{\sigma_{v}}, \chi_{I m}^{\sigma_{v}}\right)=( \pm 1, \mp 1)$ in pairs. When $H_{M M^{\prime}}$ is real, $\chi_{I m}^{\sigma_{v}}$ is chosen to be 0 . In $C_{n h}$ symmetry, $H_{M M^{\prime}}$ needs to have a parity $\left(\chi^{I / \sigma}= \pm 1\right)$ that is the product of the parities of $|M\rangle$ and $\left|M^{\prime}\right\rangle$. For an odd $n$, the parity is with respect to $\hat{\sigma}_{h}$ (' and "). For an even $n$, the parity is conventionally chosen to be with respect to $\hat{I}(g$ and $u)$. $D_{n h}$ is a composite of $D_{n}$ (which is isomorphic with $C_{n v} ;\left(\chi_{R e}^{\sigma_{v}}, \chi_{I m}^{\sigma_{v}}\right)$ replaced by $\left(\chi_{R e}^{C_{2}^{\prime}}, \chi_{I m}^{C_{2}^{\prime}}\right)$ ) and $C_{n h}$. The matrix elements of $D_{n h}(\mathrm{p}) \mathrm{JT}$ Hamiltonians are hence characterized by all 4 eigenvalues: $\left(\chi^{C_{n}},\left(\chi_{R e}^{C_{2}^{\prime}}, \chi_{I m}^{C_{2}^{\prime}}\right), \chi^{I / \sigma_{h}}\right)$.

Guided by the eigenvalues of symmetry operators, the derivation of (p)JT Hamiltonians in any of the $n$-gonal axial symmetries parallels the present derivation in tetragonal symmetry. The resultant formalism will also consist of a few lookup tables. It will be a programmable formalism. Please recall that for an odd $n, D_{n d}$ is isomorphic with $D_{n h}, D_{2 n}$ and $C_{2 n v}$; for an even $n, D_{n d}$ is only isomorphic with $D_{2 n}$ and $C_{2 n v}$. Also, for an even $n, S_{n}$ is isomorphic with $C_{n}$. 


\section{CONCLUSIONS}

In this paper, we present a formalism for all bimodal tetragonal (pseudo-)Jahn-Teller Hamiltonians. All tetragonal vibronic Hamiltonian matrix elements are characterized by at most 4 eigenvalues of symmetry operators, $\left(\chi^{C_{4}},\left(\chi_{R e}^{\sigma_{v} / C_{2}^{\prime}}, \chi_{I m}^{\sigma_{v} / C_{2}^{\prime}}\right), \chi^{I}\right)$. The number of the sets of eigenvalues is tractable and all sets are exhausted in Table 1 . The derivation for the vibronic Hamiltonians has been reduced to finding expansion formulas that are eigenfunctions of symmetry operators. This remarkable reduction and the systematic root-branch and modularized approaches have enabled us to derive analytical Hamiltonian expansion formulas up to arbitrary order for all bimodal tetragonal (p)JT problems: 54 problems in each of $C_{4}$ and $S_{4}$ symmetries, 300 in each of $C_{4 v}, D_{4}$, and $D_{2 d}, 567$ in $C_{4 h}$, and 3575 in $D_{4 h}$. In comparison, only 153 problems were covered in Ref. 22 and they all belong to a specific class of $(E+A) \otimes(e+a)$ problems in trigonal symmetry. The formalism is complete and correct, as corroborated by the numerical tests and the comparisons with previously derived formulas. The formalism is also concise, as all formulas of the thousands of problems are stored in the 9 lookup tables. Several examples are given to demonstrate the easiness in using the tables to obtain needed (p)JT Hamiltonians. We want to emphasize that one may use the formalism without understanding its derivation. To directly use the formulas, the $E$ and $e$ components must follow the orientations exemplified in Figure 1. The tabulated formalism is programmable, and we are currently working in this direction. With such a program, explicit term-by-term expansions of the 5150 problems are at our finger tips with a push of a button. Considering the ubiquity and importance of tetragonal vibronic problems, the extensive applicability of the presented formalism is evident. This work has also paved an efficient way for deriving (p)JT Hamiltonians in all axial symmetries. We have shown that in the appropriately chosen electronic diabatic representation, all matrix elements of the Hamiltonians can be similarly characterized by up to 4 eigenvalues of symmetry operators, $\left(\chi^{C_{n} / S_{n}},\left(\chi_{R e}^{\sigma_{v} / C_{2}^{\prime}}, \chi_{I m}^{\sigma_{v} / C_{2}^{\prime}}\right), \chi^{I / \sigma_{h}}\right)$. The derivations of these Hamiltonians parallel the present one. A unified formalism for vibronic Hamiltonians in any axial symmetry is within reach. 


\section{ACKNOWLEDGEMENTS}

We thank Carleton University for the start-up grant (186853) and the Natural Sciences and Engineering Research Council (NSERC) of Canada for funding (RGPIN-2016-06276). We thank Mike Schmidt (Iowa State University) and Mark Gordon (Iowa State University) for their support of the GAMESS-US program package. Computations were performed on the GPC supercomputer at the SciNet HPC Consortium. SciNet is funded by: the Canada Foundation for Innovation under the auspices of Compute Canada; the Government

of Ontario; Ontario Research Fund - Research Excellence; and the University of Toronto. 
1 H. A. Jahn and E. Teller, Proc. Roy. Soc. A, 1937, 161, 220-235.

2 U. Öpik and M. H. L. Pryce, Proc. R. Soc. A, 1957, 238, 425-447.

3 I. B. Bersuker, Adv. Chem. Phys., 2016, 160, 159-208.

4 T. A. Barckholtz and T. A. Miller, Int. Rev. Phys. Chem., 1998, 17, 435-524.

5 B. E. Applegate, T. A. Barckholtz and T. A. Miller, Chem. Soc. Rev., 2003, 32, 38-49.

6 H. Köppel, in Conical intersections: electronic structure, dynamics and spectroscopy, ed. W. Domcke, D. R. Yarkony and H. Köppel, World Scientific, New Jersey, 2004, ch. 10, pp. $429-472$.

7 I. B. Bersuker, The Jahn-Teller Effect, Cambridge University Press, Cambridge, UK, 2006.

8 M. A. Halcrow, Chem. Soc. Rev., 2013, 42, 1784-1795.

9 H. Köppel, W. Domcke and L. S. Cederbaum, Adv. Chem. Phys., 1984, 57, 59-246.

10 A. Viel and W. Eisfeld, J. Chem. Phys., 2004, 120, 4603-4613.

11 W. Eisfeld and A. Viel, J. Chem. Phys., 2005, 122, 204317.

12 S. Bhattacharyya, D. Opalka, L. V. Poluyanov and W. Domcke, J. Phys. Chem. A, 2014, 118, $11962-11970$.

13 P. Mondal, D. Opalka, L. V. Poluyanov and W. Domcke, Chem. Phys., 2011, 387, 56-65.

14 P. Mondal, D. Opalka, L. V. Poluyanov and W. Domcke, J. Chem. Phys., 2012, 136, 084308.

15 S. Bhattacharyya, D. Opalka, L. V. Poluyanov and W. Domcke, J. Phys. Conf. Series, 2013, 428, 012015.

16 W. Eisfeld, O. Vieuxmaire and A. Viel, J. Chem. Phys., 2014, 140, 224109.

17 A. Weaver, D. W. Arnold, S. E. Bradforth and D. M. Neumark, J. Chem. Phys., 1991, 94, $1740-1751$.

18 Z. Shao, H. Li, S. Zhang, J. Li, Z. Dai and Y. Mo, J. Chem. Phys., 2012, 136, 064308.

19 T. Codd, M.-W. Chen, M. Roudjane, J. F. Stanton and T. A. Miller, J. Chem. Phys., 2015, 142, 184305 .

20 D. Opalka and W. Domcke, J. Chem. Phys., 2010, 132, 154108.

21 D. Opalka and W. Domcke, Chem. Phys. Lett., 2010, 494, 134-138.

22 T. Zeng and I. Seidu, Phys. Chem. Chem. Phys., 2017, 19, 11098-11110.

23 T. Zeng, R. J. Hickman, A. Kadri and I. Seidu, J. Chem. Theory Comput., 2017, 13, 5004-5018. 
24 E. P. Wigner, Group Theory, Academic Press, Inc., 1959.

25 J. S. Griffith, The Irreducible Tensor Method for Molecular Symmetry Group, Dover Publications, Inc., 2006.

26 A. Markmann, G. A. Worth and L. S. Cederbaum, J. Chem. Phys., 2005, 122, 144320.

27 A. M. Schulenburg and F. Merkt, J. Chem. Phys., 2009, 130, 034308.

28 M.-C. Liu, S.-C. Chen, C.-H. Chin, T.-P. Huang, H.-F. Chen and Y.-J. Wu, J. Phys. Chem. Lett., 2015, 6, 3185-3189.

29 Y. Liu, I. B. Bersuker, P. Garcia-Fernandez and J. E. Boggs, J. Phys. Chem. A, 2012, 116, $7564-7570$.

30 Y. Liu, Y. Wang and I. B. Bersuker, Sci. Rep., 2016, 6, 23315.

31 A. D. Liehr, J. Phys. Chem., 1963, 67, 389-471.

32 L. V. Poluyanov and W. Domcke, Chem. Phys., 2012, 407, 1-8.

33 A. D. Becke, Phys. Rev. A, 1988, 38, 3098-3100.

34 C. Lee, W. Yang and R. G. Parr, Phys. Rev. B, 1988, 37, 785-789.

35 T. H. Dunning, J. Chem. Phys., 1989, 90, 1007-1023.

36 M. W. Schmidt, K. K. Baldridge, J. A. Boatz, S. T. Elbert, M. S. Gordon, J. H. Jensen, S. Koseki, N. Matsunaga, K. A. Nguyen, S. Su, T. L. Windus, M. Dupuis and J. A. Montgomery Jr., J. Comput. Chem., 1993, 14, 1347-1363. 